\title{
Mathematical review on source-type diagrams
}

\author{
Naofumi Aso ${ }^{1 *} \mathbb{D}$, Kazuaki Ohta ${ }^{2}$ and Satoshi Ide ${ }^{3}$
}

\begin{abstract}
A source-type diagram is a visualization tool used to display earthquake sources, including double-couples, compensated linear vector dipoles, and isotropic deformation. Together with recent observations of non-double-couple events in a variety of tectonic settings, it is important to be able to recognize the source type intuitively from a representative diagram. Since previous works have proposed diagrams created using a range of projections, we review these diagrams in the framework of the moment tensor eigenvalue space. For further applications, we also provide complete formulas for conversion between moment tensor representation and the coordinate system of each diagram style. Using both a global catalog and synthetic data, we discuss differences between types of diagrams and the relative effectiveness of each.
\end{abstract}

Keywords: Source-type diagram, Double-couple, Non-double-couple, Compensated linear vector dipole, Isotropic explosion and implosion, Theoretical seismology, Volcano seismology

\section{Background}

A source-type diagram is a visualization tool for displaying various types of seismic sources, including not only double-couple (DC) sources, but also compensated linear vector dipole (CLVD) (Knopoff and Randall 1970), opening cracks, and isotropic deformation (ISO), onto a common two-dimensional surface (Julian et al. 1998; Kawakatsu and Yamamoto 2007). Such diagrams are helpful for understanding the physical processes responsible for an earthquake, estimating the uncertainties of source type in terms of the estimation errors of the source inversion, and identifying the effect of model uncertainties such as an inaccurate source location or a poor velocity model.

Although we often assume a double-couple as the source mechanism of an earthquake, significant nondouble-couple components are reported in a variety of settings. We now review some topical examples. NonDC events are most commonly seen in volcanic and geothermal regions (e.g., Miller et al. 1998). Iguchi (1994)

\footnotetext{
*Correspondence: naso@caltech.edu

1 Seismological Laboratory, California Institute of Technology,

1200 E. Calif. Blvd., Pasadena, CA 91125, USA

Full list of author information is available at the end of the article
}

reported vertical dipole sources associated with a gas expansion process at Sakurajima volcano, Japan. Non-DC events were seen in microseismicity at Long Valley Caldera, California (Foulger et al. 2004). Saraò et al. (2001) reported temporal variations in the amplitudes of nonDC components immediately before eruptions at Etna, Italy. Tensile faulting was observed in geothermal areas of West Bohemia, Czech Republic (Vavryčuk 2002). At Hengill-Grensdalur, Iceland, Julian et al. (1997) reported combined tensile and shear faulting on vertical faults at a divergent boundary. In volcanic and geothermal regions, such small-scale non-DC sources are likely related to phase changes of volatiles in magma or groundwater. A non-DC component has also been found in some deep volcanic long-period events (volcanic DLPs) that occur around the Moho; most of these were beneath active volcanoes, but some did not correspond to any known volcano with Quaternary activity (Nishidomi and Takeo 1996; Okada and Hasegawa 2000; Nakamichi et al. 2003; Aso and Ide 2014). Thermal contraction of a cooling magma body has been suggested as a possible source for the non-DC component of these events (Aso and Tsai 2014). Using regional broadband records, Legrand et al. (2000) observed long-period ( 10 s) isotropic deformation events at Aso, Japan. Non-DC components have also 
been observed geodetically at Miyakejima, Japan (Minson et al. 2007). From a statistical analysis of a global catalog of larger events, Shuler et al. (2013) showed that vertical CLVDs are often observed at volcanoes worldwide. These larger-scale non-DC events might be related to long-term volcanic evolutionary processes, including caldera formation (e.g., Ekström 1994). Non-DC source mechanisms have also been reported in induced seismicity. For example, non-DC events have been observed among earthquakes induced by wastewater injection in Geysers, California (Ross et al. 1996, 1999) and southeast Germany (Vavryčuk et al. 2008). Mining-induced earthquakes (e.g., Ford et al. 2008) are also reported to have non-DC mechanisms.

Under the right conditions, non-DC sources can be seen on more typical faults. Distributed shear slip along a finite bending fault appears to produce a CLVD component when regarded as a point source; this has been demonstrated by moment tensor inversions that allow temporal variation (Sipkin 1986) and spatiotemporal variation (Kikuchi et al. 1993). Considering that an earthquake fault consists of many subfaults, the relationship between the CLVD component and the seismic moment could reflect scale-dependent fault zone irregularities (Kuge and Lay 1994b). Planar shear faulting in anisotropic media can also produce a non-DC component (Vavryčuk 2005). The possibility of an isotropic component for deep-focus earthquakes, first suggested by Dziewonski and Gilbert (1974), has been a controversial topic. However, while the existence of the isotropic component is still debated (e.g., Okal and Geller 1979), the existence of a CLVD component related to subevents is strongly supported for deep-focus earthquakes (Kuge and Kawakatsu 1990). Tensile mechanisms have been found for icequakes at Gornergletscher, Switzerland (Walter et al. 2009). Apart from natural earthquakes, man-made events such as nuclear explosions (e.g., Ford et al. 2009) are notable examples of isotropic events. Since this variety of earthquakes comprises a wide range of source mechanisms, it is important to use interpretable visual diagrams to compare and contrast.

Although non-DC events have been reported under various conditions, some non-DC components in previous works might be artifacts of using inappropriate assumptions in the inversion process (e.g., Adamová and Šílený 2010), such as near-source heterogeneity (Kuge and Kawakatsu 1993). The reliability of the non-DC component also depends on the type of seismic phase data used, i.e., body waves, surface waves, or normal modes (Kuge and Lay 1994a; Hara et al. 1995). An isotropic component is difficult to resolve from teleseismic data alone (Dufumier and Rivera 1997). Although dislocation on a bimaterial surface can be expressed by a moment tensor, its accurate estimation is difficult because the Green's function calculation must consider the interface geometry correctly (Ampuero and Dahlen 2005; Vavryčuk 2013). Furthermore, since events driven by a single force cannot be expressed by a moment tensor (Kawakatsu 1989), moment tensor representations of such events result in artificial solutions. Source-type diagrams can be used to visualize the potential bias of source-type solutions from each analysis (e.g., Ford et al. 2010).

The most commonly used method to visualize source types is the representation of Hudson et al. (1989). Recently, Tape and Tape (2012) clarified this projection mathematically, based on geometrical concepts introduced by Chapman and Leaney (2012). Various other diagrams have developed over time, using different perspectives to represent an earthquake source (Chapman and Leaney 2012; Zhu and Ben-Zion 2013; Vavryčuk 2015). Therefore, this paper provides a comprehensive review and classification of these diagram styles, based on the geometrical framework of Chapman and Leaney (2012) and Tape and Tape (2012). As we will show, different diagrams are useful for different purposes, just as with map projections; we therefore present case-by-case suggestions for appropriate usage in future studies.

Although previous studies that introduced new sourcetype diagrams are each mathematically complete, simple conversion formulas from a moment tensor to the coordinates of different diagrams are lacking. In this study, we focus on source-type diagrams and provide a complete formula for producing each diagram. Simple conversion formulas from a moment tensor to each diagram type (or, equivalently, to each projection's coordinate system) are given in the main text for further applications. Derivations are presented in the Appendices.

Inverse conversion, from source-type coordinates to moment tensor representation, is valuable for random search (e.g., Ford et al. 2010) and grid search algorithms (e.g., Aso and Ide 2014; Nayak and Dreger 2015), which find a moment tensor that best fits a seismogram. Selecting various points in the source-type diagram, and converting them into moment tensors, will facilitate systematic and efficient searches over a possible range of source types.

In the present study, we first review various diagram types and conversion formulas. While most of these originated in previous works, we also suggest some new alternative projections with advantageous properties. Finally, we explore differences between diagram styles using real and synthetic moment tensor catalog.

\section{Mathematical formulations of source-type diagrams}

The equivalent body forces needed to produce deformation in a small volume of the Earth's interior are usually 
expressed as a symmetric moment tensor located at a point in space. The $3 \times 3$ symmetric tensor (six degrees of freedom) is converted to three real eigenvalues (three degrees of freedom) and three orthogonal unit eigenvectors (three degrees of freedom). The eigenvectors contain information about the characteristic orientation of the source, while the eigenvalues represent a scalar source amplitude (size) and a source type. Since source type has two degrees of freedom, its visual representation can be projected onto a two-dimensional plane. Various approaches can be used to separate the size and source type from the eigenvalues, depending on how the norm (size) of the moment tensor is defined. We describe moment tensors using a three-dimensional Cartesian coordinate system $\left(\lambda_{1}, \lambda_{2}, \lambda_{3}\right)$, where each $\lambda$ represents one eigenvalue of the moment tensor $M_{i j}$. We will refer to the space defined by $\left(\lambda_{1}, \lambda_{2}, \lambda_{3}\right)$ as the moment tensor eigenvalue space. We note that this does not meet the strict mathematical definition of an eigenspace; instead, we term it an "eigenvalue space." The angle between two source mechanisms $\mathrm{A}$ and $\mathrm{B}$ in the eigenvalue space, $\cos ^{-1}\left(\sum_{i} \lambda_{i}^{A} \lambda_{i}^{B} / \sqrt{\sum_{i} \lambda_{i}^{A} \lambda_{i}^{A}} \sqrt{\sum_{i} \lambda_{i}^{B} \lambda_{i}^{B}}\right)$, is equivalent to the tensor angle in the moment tensor space, $\cos ^{-1}\left(\sum_{i j} M_{i j}^{A} M_{i j}^{B} / \sqrt{\sum_{i j} M_{i j}^{A} M_{i j}^{A}} \sqrt{\sum_{i j} M_{i j}^{B} M_{i j}^{B}}\right)$, provided that these mechanisms share common eigenvectors; therefore, the eigenvalue space is suitable for recognizing the similarity of two moment tensors. Since changing the order of the eigenvalues does not correspond to a different physical source, but instead corresponds to a different selection of eigenvectors, we can order the eigenvalues $\lambda_{1} \geq \lambda_{2} \geq \lambda_{3}$ without loss of generality. With this ordering, all mechanisms fall into onesixth of the whole three-dimensional moment tensor eigenvalue space. In Fig. 1, this region is shown in green, enclosed by a blue line $\left(\lambda_{1}=\lambda_{2}\right)$ and a red line $\left(\lambda_{2}=\lambda_{3}\right)$.

We first consider the locations of the three end-member source mechanism types in the moment tensor eigenvalue space: double-couple (DC), compensated linear vector dipole (CLVD), and isotropic (ISO). Although an opening crack is sometimes used as a more physically realistic alternative to pure CLVD, the moment tensor of an opening crack depends on the Poisson's ratio, unlike that of the CLVD itself. Hence, we use a CLVD end member in this work to guarantee a strictly mathematical framework. We express an explosive source as +ISO and an implosive source as -ISO. We use + CLVD to denote a CLVD whose polarity along the axis of symmetry is positive, and -CLVD for the opposite. On the mesh grid in Fig. 1a, which lies on the cubic surface of $\max \left(\left|\lambda_{1}\right|,\left|\lambda_{2}\right|,\left|\lambda_{3}\right|\right)=1$, these end members are represented by black points: \pm ISO on $\pm(1,1,1)$, DC on $(1,0,-1)$, and \pm CLVD on $(1,-1 / 2,-1 / 2)$ and $(1 / 2,1 / 2,-1)$. We note that not only these points correspond to those end-member mechanisms, since they are distributed in three dimensions. For example, all points $\propto(1,0,-1)$ on a line in this space correspond to a DC source.

Once we define a norm in the moment tensor eigenvalue space, points in the three-dimensional space can be projected onto a two-dimensional surface of unit "size" $(A=1)$; this is the basic process of producing a source-type diagram. One definition of a norm is a

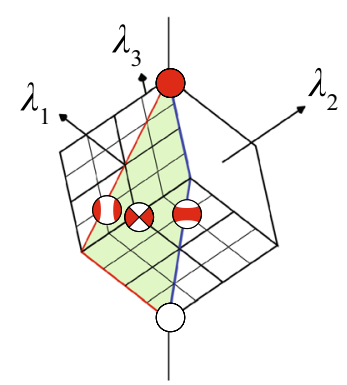

b

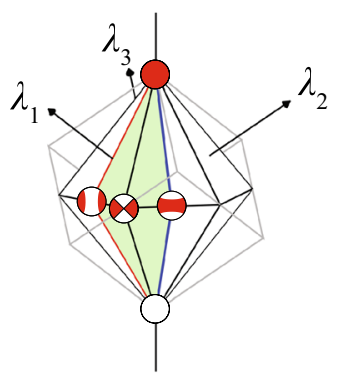

C

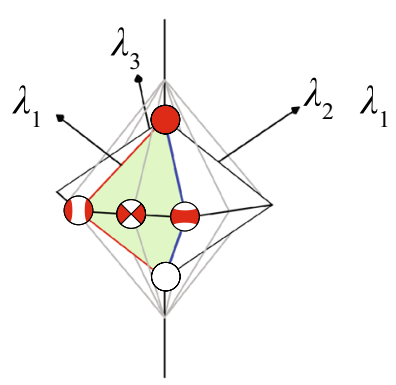

d

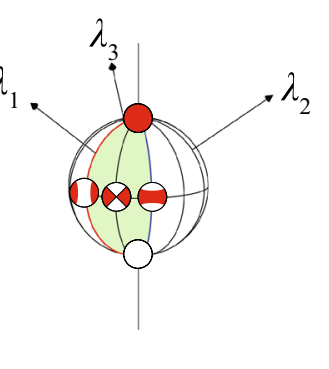

Fig. 1 Moment tensor eigenvalue space and basal projections. Graphical representation of the moment tensor eigenvalue space $\left(\lambda_{1}, \lambda_{2}, \lambda_{3}\right)$. The blue line corresponds to the condition $\lambda_{1}=\lambda_{2}$, and the red line corresponds to the condition $\lambda_{2}=\lambda_{3}$. The green region represents the condition $\lambda_{1} \geq \lambda_{2} \geq \lambda_{3}$. Locations of DC, \pm CLVD, and \pm ISO source types on the surface are shown by their corresponding beach-ball symbols. $\mathbf{a}$ In the basal cubic projection, all mechanisms are projected onto two triangles on the cube defined by $\max \left(\left|\lambda_{1}\right|,\left|\lambda_{2}\right|,\left|\lambda_{3}\right|\right)=1$. The subgrid interval is 0.5 . $\mathbf{b}$ In the basal hexagonal bi-pyramid projection, all mechanisms are projected onto four triangles on the hexagonal bi-pyramid that fits the cube of a, which is shown in gray. $\mathbf{c}$ In the basal conjugate hexagonal bi-pyramid projection, all mechanisms are projected onto the two triangles on the hexagonal bi-pyramid. The hexagonal bi-pyramid of $\mathbf{b}$ is shown in gray. $\mathbf{d}$ In the basal spherical projection, all mechanisms are projected onto a lune on the sphere 
the absolute maximum amplitude of the eigenvalues, $A_{c}=\max \left(\left|\lambda_{1}\right|,\left|\lambda_{2}\right|,\left|\lambda_{3}\right|\right)$, whose corresponding unit surface is a cube (Fig. 1a). This size is proportional to the maximum amplitude of $\mathrm{P}$-wave radiation for point sources in an isotropic medium that share a common source time function. We can consider other projections on a hexagonal bi-pyramid (Fig. 1b) or its conjugate shape (Fig. 1c), although the seismological meanings of their size definitions are not as clear. Another size definition is the Cartesian norm, $A_{s}=\sqrt{\lambda_{1}^{2}+\lambda_{2}^{2}+\lambda_{3}^{2}}$, whose corresponding unit surface is a sphere (Fig. 1d). This is proportional to the scalar moment, $M_{o}=\sqrt{\left(\lambda_{1}^{2}+\lambda_{2}^{2}+\lambda_{3}^{2}\right) / 2}$ (Bowers and Hudson 1999; Shearer 2009). Strictly speaking, the only diagram that maintains angles between two mechanisms is the spherical projection, but we will consider various projections of the eigenvalue space nonetheless. This is warranted because even the curved surface of the spherical projection is converted to a flat surface later; thus, the angle between two mechanisms is not strictly preserved in the final two-dimensional visualization.

Before explaining the details of each diagram, we first clarify the notation used. Since the horizontal and vertical scales differ among various diagrams, we suggest normalization of coordinates to facilitate direct comparisons. The equidimensional shape of a normalized diagram also makes it easier to use; some original diagram styles are vertically extended. In most of the original works, -CLVD is located on the rightmost end point and +CLVD on the leftmost end point, yet as pointed out by Chapman and Leaney (2012), this is not intuitive. Since we prefer to have +CLVD or tensile cracks on the right-hand side of the diagram for maximum usability, we reflect diagrams about the vertical axis during the normalization process. The corresponding mathematical operation is written as a negative normalization factor applied to the horizontal coordinate. The notation and normalization for each diagram are given in Table 1.

In the following subsections, we categorize sourcetype diagrams by norm. We explain their historical backgrounds and relationships with their three-dimensional parent surfaces and derive formulas for conversion from moment tensor representation. We provide forward conversions from eigenvalues to source-type coordinates for all diagrams in the text. Please refer to the Appendices for detailed derivations, including all inverse conversion formulas.

\section{Projection onto a cube}

The pioneering study of Hudson et al. (1989) produced a source-type diagram merely by projection onto an open-face cube (Fig. 2a). Henceforth, we call this a "cubic projection" and refer to it with the letter (a). Following Chapman and Leaney (2012), Tape and Tape (2012), and Vavryčuk (2015), the conversion from eigenvalues to $u-v$ coordinates is given by

Table 1 Coordinates of source-type diagrams

\begin{tabular}{|c|c|c|c|c|c|}
\hline & & Horizontal axis & Vertical axis & Normalization & Reference \\
\hline (a) & Cubic & $u$ & $v$ & $(-u, v)$ & Hudson et al. (1989) \\
\hline (b) & Hexagonal bi-pyramid & $\tau$ & k & $(-\tau, k)$ & $\begin{array}{l}\text { Hudson et al. (1989) } \\
\text { Vavryčuk (2015) }\end{array}$ \\
\hline (c) & Modified hexagonal bi-pyramid & $T=\frac{\tau}{1-|k|}$ & k & $(-T, k)$ & $\begin{array}{l}\text { Hudson et al. (1989) } \\
\text { Vavryčuk (2015) }\end{array}$ \\
\hline (d) & Conjugate bi-pyramid & $\eta$ & $\xi$ & $(-\eta, \xi)$ & Vavryčuk (2015) \\
\hline (e) & Spherical equirectangular & $\gamma$ & $\delta$ & $\left(-\frac{6 \gamma}{\pi}, \frac{2 \delta}{\pi}\right)$ & $\begin{array}{l}\text { Chapman and Leaney (2012) } \\
\text { Tape and Tape (2012) }\end{array}$ \\
\hline$(f)$ & Spherical orthogonal & $R=\sin \gamma \cos \delta$ & $\zeta=\sin \delta$ & $(-2 R, \zeta)$ & - \\
\hline (g) & Modified spherical orthogonal & $r=R|R|$ & $s=\zeta|\zeta|$ & $(-4 r, s)$ & $\begin{array}{l}\text { Zhu and Ben-Zion (2013) } \\
\text { Vavryčuk (2015) }\end{array}$ \\
\hline (h) & Spherical azimuthal & $p=\frac{\sqrt{2} \sin \gamma \cos \delta}{\sqrt{1+\cos \gamma \cos \delta}}$ & $q=\frac{\sqrt{2} \sin \delta}{\sqrt{1+\cos \gamma \cos \delta}}$ & $\left(\frac{-2 p}{\sqrt{6}-\sqrt{2}}, \frac{q}{\sqrt{2}}\right)$ & $\begin{array}{l}\text { Chapman and Leaney (2012) } \\
\text { Tape and Tape (2013) } \\
\text { Vavryčuk (2015) }\end{array}$ \\
\hline (i) & Spherical cylindrical & $\gamma$ & $\zeta$ & $\left(-\frac{6 \gamma}{\pi}, \zeta\right)$ & Tape and Tape (2012) \\
\hline$(j)$ & Modified spherical cylindrical & $a=\frac{6}{\pi} \gamma \sqrt{1-|\zeta|}$ & $b=\frac{\zeta}{1+\sqrt{1-|\zeta|}}$ & $(-a, b)$ & - \\
\hline$(k)$ & Spherical cylindrical orthogonal & $\chi=\sin \gamma$ & $\zeta$ & $(-2 \chi, \zeta)$ & Zhu and Ben-Zion (2013) \\
\hline (l) & Percentile & $\epsilon$ & $v$ & $(-2 \epsilon, v)$ & Vavryčuk (2001) \\
\hline$(\mathrm{m})$ & Modified percentile & $c=2 \epsilon(1-|v|)$ & v & $(-c, v)$ & Vavryčuk (2001) \\
\hline
\end{tabular}


a Cubic

$(u-v)$
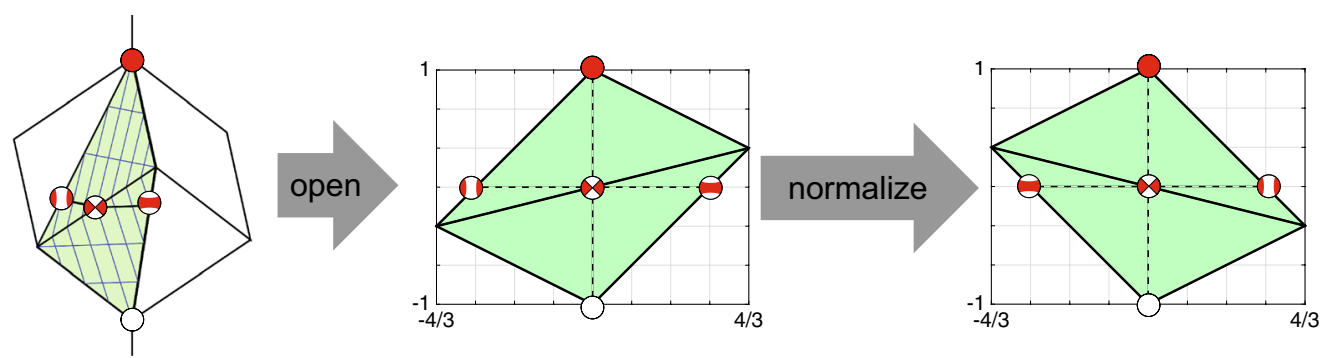

b Hexagonal Bi-Pyramid

$(\tau-k)$

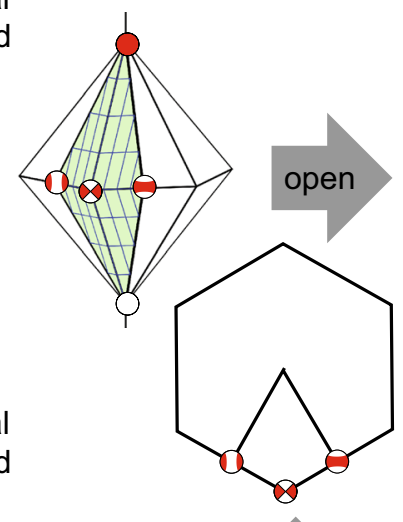

c Modified

Hexagonal

Bi-Pyramid

$(T-k)$
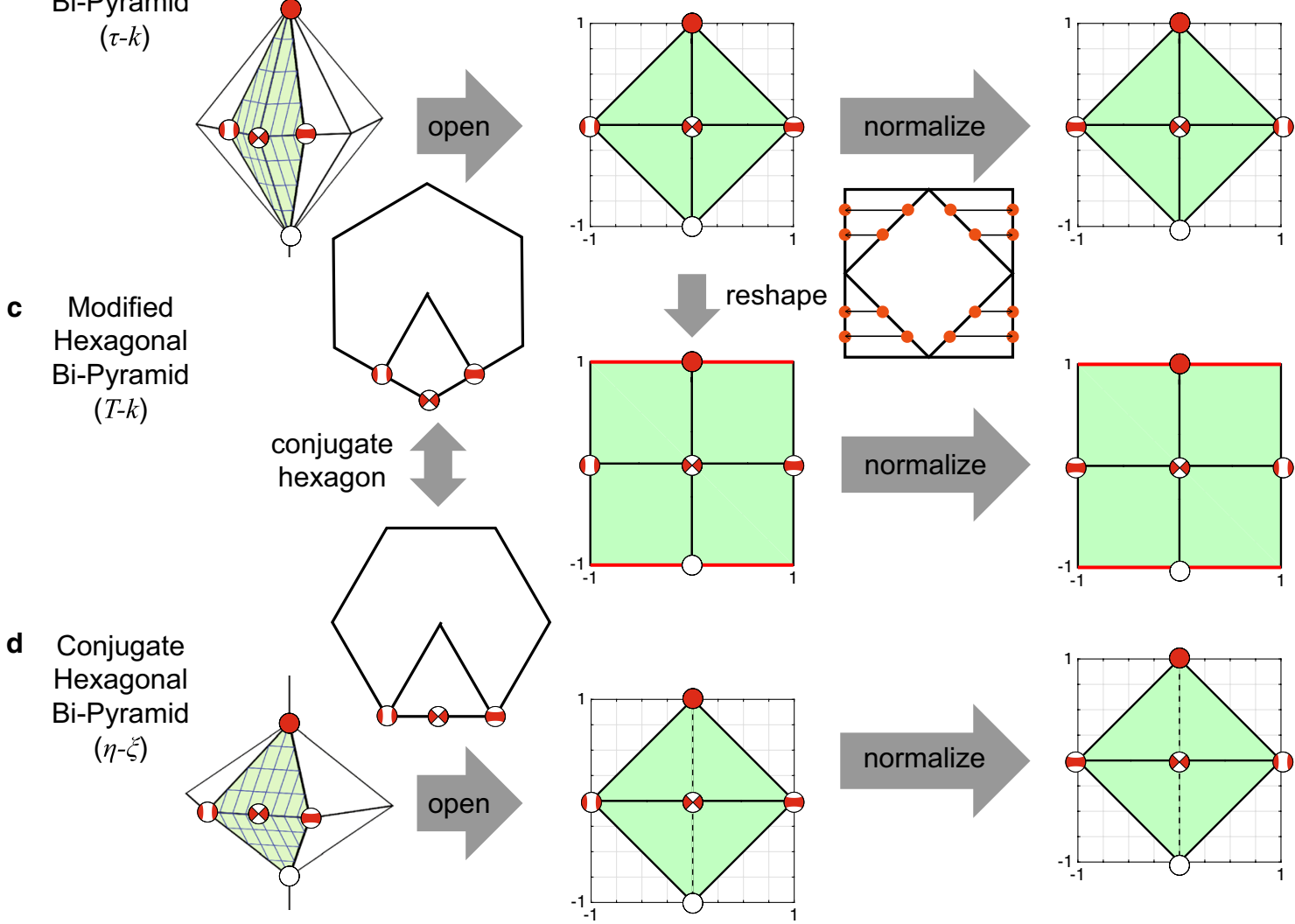

Fig. 2 Projections to produce source-type diagrams. Steps to produce each source-type diagram using each projection are illustrated in sequential order. The original source-type diagram (middle column) and the source-type diagram after normalization (right column) are shown for each projection. For most diagrams, the corresponding surface in the moment tensor eigenvalue space is also shown (left column). In each 2D source-type diagram, the projected area is shown in green; solid lines represent sharp interfaces, across which a trace of a gradual change in the moment tensor can generate a curve with a cusp. a Cubic projection diagrams $(u, v)$ and $(\tilde{u}, \tilde{v})$. b Hexagonal bi-pyramid projection diagrams $(\tau, k)$ and $(\tilde{\tau}, \tilde{k})$. c Modified hexagonal bi-pyramid projection diagrams $(T, k)$ and $(\tilde{T}, \tilde{k})$. d Conjugate hexagonal bi-pyramid projection $\operatorname{diagrams}(\eta, \xi)$ and $(\tilde{\eta}, \tilde{\xi})$

$$
\left[\begin{array}{l}
u \\
v
\end{array}\right]=\left[\begin{array}{c}
\frac{-2\left(\lambda_{1}-2 \lambda_{2}+\lambda_{3}\right)}{3 \max \left(\lambda_{1},-\lambda_{3}\right)} \\
\frac{\lambda_{1}+\lambda_{2}+\lambda_{3}}{3 \max \left(\lambda_{1},-\lambda_{3}\right)}
\end{array}\right] .
$$

Detailed derivation, including the inverse conversion formula, is provided in "Appendix 1." To align the +CLVD term on the right-hand side of the plot, we introduce a sign reversal, $(\tilde{u}, \tilde{v})=(-u, v)$, during normalization. Although this diagram is popular, its oblique quadrant (or skewed rectangle) shape makes it slightly difficult to use. In particular, the corners of this diagram do not correspond to \pm CLVD and have less physical or mathematical meaning than \pm CLVD. 


\section{Projection onto a hexagonal bi-pyramid}

Hudson et al. (1989) introduced another projection called the $\tau-k$ plot. This square diamond shape is created by projection onto an open-face hexagonal bi-pyramid (Fig. 2b); it shares all the surfaces of the cubic projection described above. The second and forth quadrants of this projection are identical to those of the cubic projection. We refer to this diagram herein as a "hexagonal bi-pyramid projection" (b). This diagram is termed the "standard decomposition" in Vavryčuk (2015). The conversion from eigenvalues to $\tau-k$ coordinates is given by

$$
\left[\begin{array}{l}
\tau \\
k
\end{array}\right]=\left[\begin{array}{c}
\frac{-4\left(\lambda_{1}-2 \lambda_{2}+\lambda_{3}\right)}{3\left(\lambda_{1}-\lambda_{3}\right)+\left|\lambda_{1}-2 \lambda_{2}+\lambda_{3}\right|+2\left|\lambda_{1}+\lambda_{2}+\lambda_{3}\right|} \\
\frac{2\left(\lambda_{1}+\lambda_{2}+\lambda_{3}\right)}{3\left(\lambda_{1}-\lambda_{3}\right)+\left|\lambda_{1}-2 \lambda_{2}+\lambda_{3}\right|+2\left|\lambda_{1}+\lambda_{2}+\lambda_{3}\right|}
\end{array}\right] .
$$

Here, the sign reversal that aligns the +CLVD axis on the right-hand side is $(\tilde{\tau}, \tilde{k})=(-\tau, k)$. Full details are provided in "Appendix 2."

Based on this transformation, a diagram with a regular square shape can be produced by extending the horizontal range (Fig. 2c). This "modified hexagonal bi-pyramid projection" (c) is found in Hudson et al. (1989) under the name " $T-k$ space," and is given by

$$
\left[\begin{array}{l}
T \\
k
\end{array}\right]=\left[\begin{array}{c}
\frac{-4\left(\lambda_{1}-2 \lambda_{2}+\lambda_{3}\right)}{3\left(\lambda_{1}-\lambda_{3}+\left|\lambda_{1}-2 \lambda_{2}+\lambda_{3}\right|\right.} \\
\frac{2\left(\lambda_{1}+\lambda_{2}+\lambda_{3}\right)}{3\left(\lambda_{1}-\lambda_{3}\right)+\left|\lambda_{1}-2 \lambda_{2}+\lambda_{3}\right|+2\left|\lambda_{1}+\lambda_{2}+\lambda_{3}\right|}
\end{array}\right] .
$$

Details of this projection appear in "Appendix 3." Similar to (1) and (2), we introduce the sign reversal $(\tilde{T}, \tilde{k})=(-T, k)$ during normalization.

If we rotate the hexagonal bi-pyramid around the vertical axis by $\pi / 6$ radians, we can define a projection of the three-dimensional space onto two surfaces (Fig. 2d). Note that the height of the conjugate hexagonal bi-pyramid can be set arbitrarily. After modification of the height from the original hexagonal bi-pyramid, we get the "simplified decomposition" projection of Vavryčuk (2015), which can indeed be described by a simple formula; this conjugate hexagonal bi-pyramid projection (d) is given by

$$
\left[\begin{array}{l}
\eta \\
\xi
\end{array}\right]=\left[\begin{array}{c}
\frac{-\left(\lambda_{1}-2 \lambda_{2}+\lambda_{3}\right)}{\lambda_{1}-\lambda_{3}+\left|\lambda_{1}+\lambda_{2}+\lambda_{3}\right|} \\
\frac{\lambda_{1}+\lambda_{2}+\lambda_{3}}{\lambda_{1}-\lambda_{3}+\left|\lambda_{1}+\lambda_{2}+\lambda_{3}\right|}
\end{array}\right] .
$$

Details of this projection are provided in "Appendix 4." To plot +CLVD on the right-hand side of the diagram, the appropriate sign reversal is $(\tilde{\eta}, \tilde{\xi})=(-\eta, \xi)$.

\section{Projection onto a sphere}

Projection onto a sphere is reasonably consistent with the idea of a moment tensor, but the obtained two-dimensional surface is not flat, and additional projection(s) must be applied to create a flat (two-dimensional) representation.

One simple way to create a flat surface is to use an orthogonal polar coordinate system on a lune; e.g., latitude and longitude (Fig. 3e). These coordinates were originally introduced by Tape and Tape (2012), although they did not intend to produce this diagram. The "spherical equirectangular projection" (e) is written

$$
\left[\begin{array}{l}
\gamma \\
\delta
\end{array}\right]=\left[\begin{array}{c}
-\tan ^{-1}\left(\frac{\lambda_{1}-2 \lambda_{2}+\lambda_{3}}{\sqrt{3}\left(\lambda_{1}-\lambda_{3}\right)}\right) \\
\sin ^{-1}\left(\frac{\lambda_{1}+\lambda_{2}+\lambda_{3}}{\sqrt{3\left(\lambda_{1}^{2}+\lambda_{2}^{2}+\lambda_{3}^{2}\right)}}\right)
\end{array}\right] .
$$

To facilitate comparison with other diagrams, we apply normalization and a sign reversal, given by $(\tilde{\gamma}, \tilde{\delta})=(-6 \gamma / \pi, 2 \delta / \pi)$. Further details appear in "Appendix 5."

A basic geometric projection of a three-dimensional lune is the orthogonal projection shown in Fig. 3f, where the lune is projected onto an oblique circle. Although no previous study has used this diagram, the orthogonal projection is a simple idea; this is the basal diagram that produces the diagram used by Zhu and Ben-Zion (2013). This "spherical orthogonal projection" (f) ("Appendix 6" section) is given by

$$
\left[\begin{array}{l}
R \\
\zeta
\end{array}\right]=\left[\begin{array}{c}
\frac{-\left(\lambda_{1}-2 \lambda_{2}+\lambda_{3}\right)}{\sqrt{6\left(\lambda_{1}^{2}+\lambda_{2}^{2}+\lambda_{3}^{2}\right)}} \\
\frac{\lambda_{1}+\lambda_{2}+\lambda_{3}}{\sqrt{3\left(\lambda_{1}^{2}+\lambda_{2}^{2}+\lambda_{3}^{2}\right)}}
\end{array}\right] .
$$

Considering that the aspect ratio of the oblique circle is two, we introduce the normalization $(\tilde{R}, \tilde{\zeta})=(-2 R, \zeta)$.

Based on the spherical orthogonal projection, a diamond-shaped diagram can be produced by taking the square of each coordinate (Fig. 3g); such a diagram was first described by Zhu and Ben-Zion (2013). This "modified spherical orthogonal projection" ( $\mathrm{g}$ ) is given by

$$
\left[\begin{array}{l}
r \\
s
\end{array}\right]=\left[\begin{array}{c}
\frac{-\left(\lambda_{1}-2 \lambda_{2}+\lambda_{3}\right)\left|\lambda_{1}-2 \lambda_{2}+\lambda_{3}\right|}{6\left(\lambda_{1}^{2}+\lambda_{2}^{2}+\lambda_{3}^{2}\right)} \\
\frac{\left(\lambda_{1}+\lambda_{2}+\lambda_{3}\right)\left|\lambda_{1}+\lambda_{2}+\lambda_{3}\right|}{3\left(\lambda_{1}^{2}+\lambda_{2}^{2}+\lambda_{3}^{2}\right)}
\end{array}\right] .
$$

Since its shape is vertically long, we introduce the normalization $(\tilde{r}, \tilde{s})=(-4 r, s)$ to facilitate comparison with other diagrams. Further details appear in "Appendix 7."

Another possible two-dimensional projection of a three-dimensional lune would be an azimuthal equalarea projection (Fig. 4h), conceptually similar to the "beach-ball" projection (Honda and Emura 1958). This diagram is identical to that developed by Chapman and Leaney (2012) and seems to be the same as that used by Tape and Tape (2013). This "spherical azimuthal projection" (h) can be written 


\section{e Spherical} Equirectangular $(\gamma-\delta)$
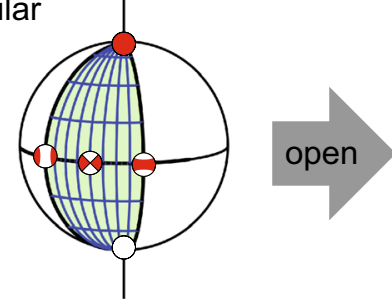

f Spherical
Orthogonal $(R-\zeta)$
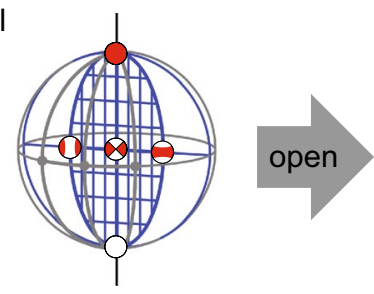

g Modified

Spherical

Orthogonal

$(r-s)$
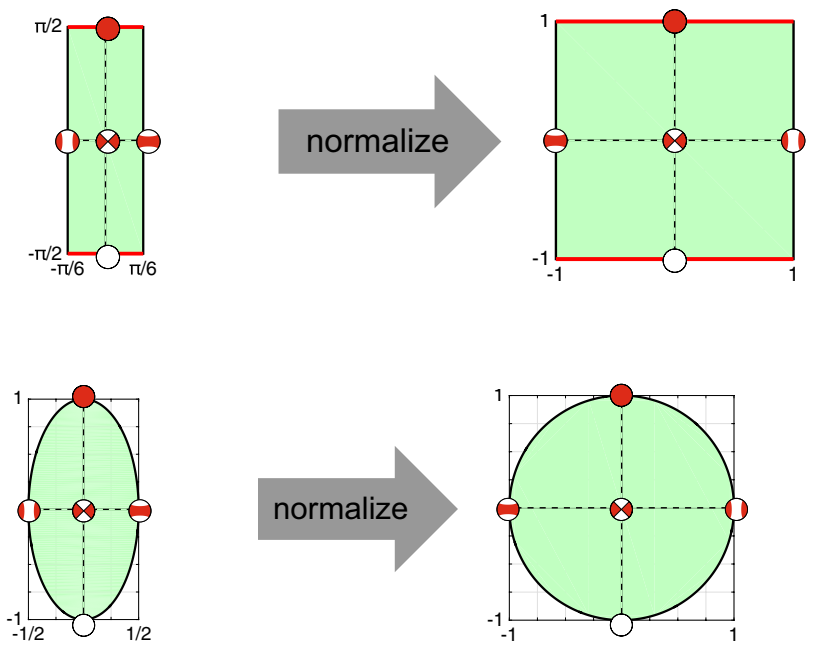

\section{normalize}
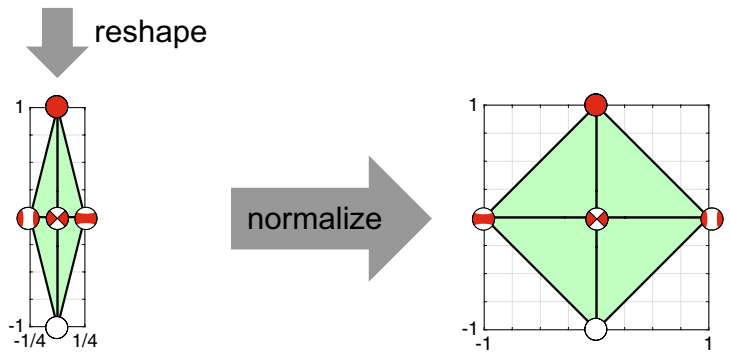

Fig. 3 Projections to produce source-type diagrams. Steps to produce each source-type diagram using each projection are illustrated in sequential order. The original source-type diagram (middle column) and the source-type diagram after normalization (right column) are shown for each projection. For most diagrams, the corresponding surface in the moment tensor eigenvalue space is also shown (left column). In each 2D source-type diagram, the projected area is shown in green; solid lines represent sharp interfaces, across which a trace of a gradual change in the moment tensor can generate a curve with a cusp. e Spherical equirectangular projection diagrams $(\gamma, \delta)$ and $(\tilde{\gamma}, \tilde{\delta})$. f Spherical orthogonal projection diagrams $(R, \zeta)$ and $(\tilde{R}, \tilde{\zeta}) \cdot \mathbf{g}$ Modified spherical orthogonal projection diagrams $(r, s)$ and $(\tilde{r}, \tilde{\zeta})$

$$
\left[\begin{array}{c}
p \\
q
\end{array}\right]=\frac{1}{\sqrt{3}}\left[\frac{\frac{-\left(\lambda_{1}-2 \lambda_{2}+\lambda_{3}\right)}{\sqrt{\lambda_{1}^{2}+\lambda_{2}^{2}+\lambda_{3}^{2}+\left(\lambda_{1}-\lambda_{3}\right) \sqrt{\left(\lambda_{1}^{2}+\lambda_{2}^{2}+\lambda_{3}^{2}\right) / 2}}}}{\sqrt{\lambda_{1}^{2}+\lambda_{2}^{2}+\lambda_{3}^{2}+\left(\lambda_{1}-\lambda_{3}\right) \sqrt{\left(\lambda_{1}^{2}+\lambda_{2}^{2}+\lambda_{3}^{2}\right) / 2}}}\right]
$$

Full details of this projection appear in "Appendix 8." Since the range of values is $|p| \leq(\sqrt{6}-\sqrt{2}) / 2,|q| \leq \sqrt{2}$, and the isotropic component in the vertical direction tends to be exaggerated, whereas the deviatoric component in the horizontal direction does not, we use the axis normalization $(\tilde{p}, \tilde{q})=(-2 p /(\sqrt{6}-\sqrt{2}), q / \sqrt{2})$ to compare this projection with other diagrams. This diagram has the advantage that a straight line passing through the center corresponds to the mechanism obtained by a combination of an arbitrary mechanism with a double-couple. The main disadvantage of this diagram is its curved shape; as derived in "Appendix 8," $p$ and $q$ are related by

$$
p= \pm \frac{\sqrt{3\left(8-3 q^{2}\right)}-\sqrt{8-q^{2}}}{4} .
$$

This lemon-like shape is similar to an oblique circle, except for sharp tips at the vertical ends.

A cylindrical projection of a lune is another equal-area projection. Here, we suggest a cylindrical projection that produces an equal-area rectangular diagram (Fig. 4i). This idea was also suggested by Tape and Tape (2012), although they did not intend to use this diagram. This "spherical cylindrical projection" (i) is given by

$$
\left[\begin{array}{l}
\gamma \\
\zeta
\end{array}\right]=\left[\begin{array}{c}
-\tan ^{-1}\left(\frac{\lambda_{1}-2 \lambda_{2}+\lambda_{3}}{\sqrt{3}\left(\lambda_{1}-\lambda_{3}\right)}\right) \\
\frac{\lambda_{1}+\lambda_{2}+\lambda_{3}}{\sqrt{3\left(\lambda_{1}^{2}+\lambda_{2}^{2}+\lambda_{3}^{2}\right)}}
\end{array}\right]
$$

We introduce the normalization $(\tilde{\gamma}, \tilde{\zeta})=(-6 \gamma / \pi, \zeta)$ to facilitate comparison with the other diagrams. Full 
h Spherical Azimuthal $(p-q)$

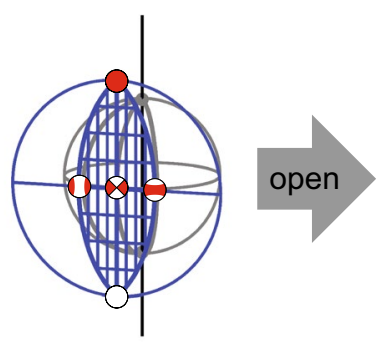

i Spherical Cylindrical $(\gamma-\zeta)$
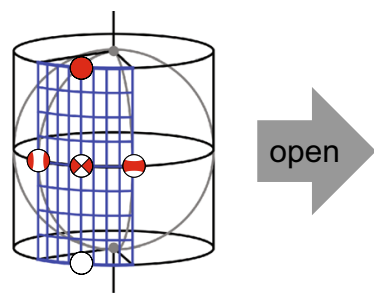

j Modified Spherical Cylindrical $(a-b)$

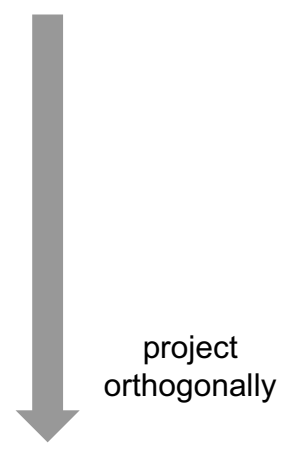

k Spherical Cylindrical Orthogonal $(\alpha-\zeta)$

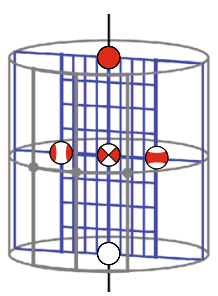

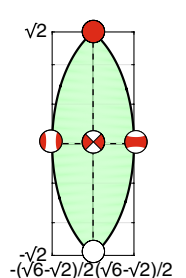

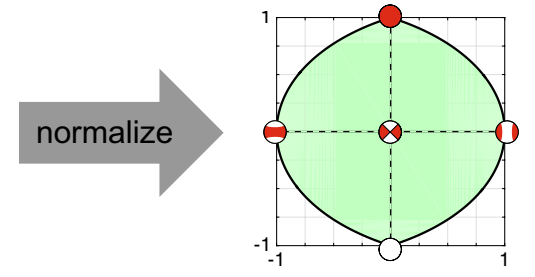

$-(\sqrt{6}-\sqrt{2}) / 2(\sqrt{6}-\sqrt{2}) / 2$

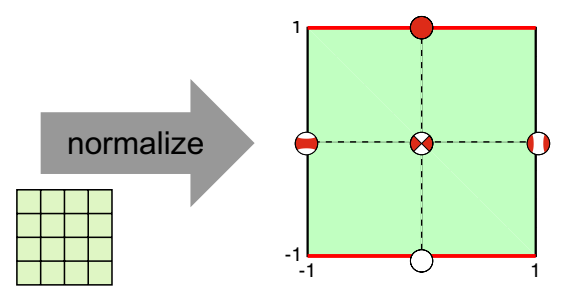

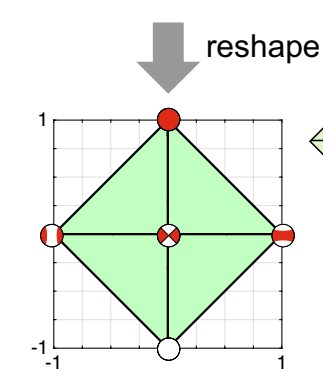
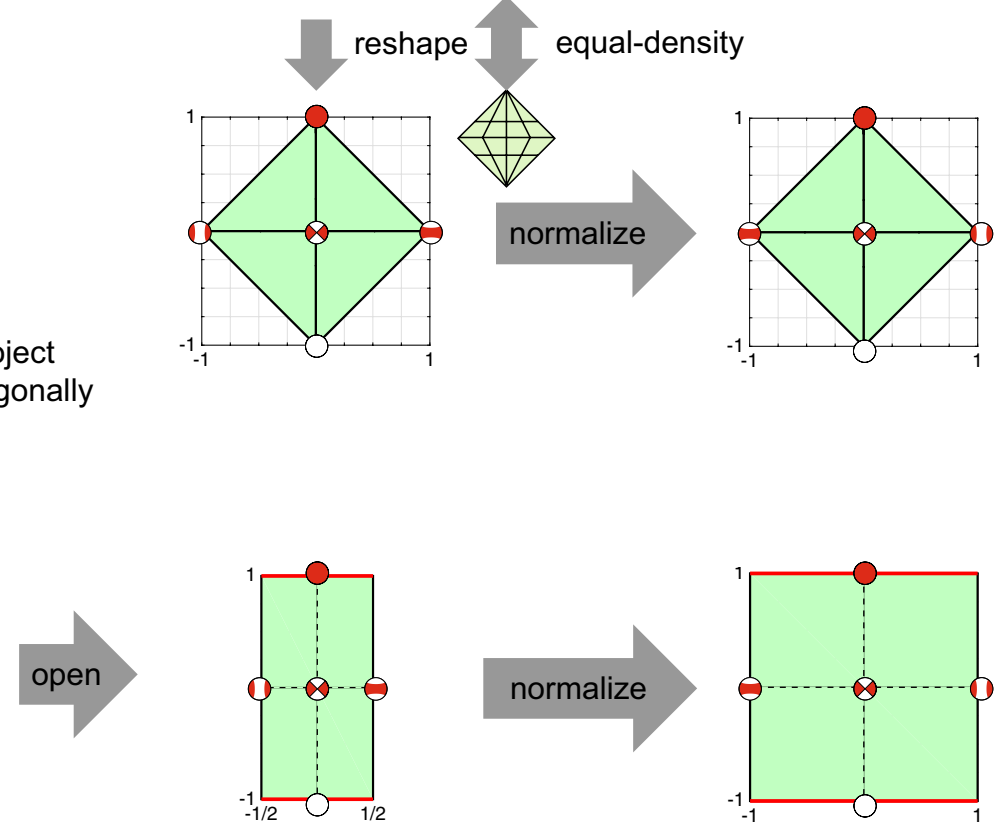

Fig. 4 Projections to produce source-type diagrams. Steps to produce each source-type diagram using each projection are illustrated in sequential order. The original source-type diagram (middle column) and the source-type diagram after normalization (right column) are shown for each projection. For most diagrams, the corresponding surface in the moment tensor eigenvalue space is also shown (left column). In each 2D source-type diagram, the projected area is shown in green; solid lines represent sharp interfaces, across which a trace of a gradual change in the moment tensor can generate a curve with a cusp. $\mathbf{h}$ Spherical azimuthal projection diagrams $(p, q)$ and $(\tilde{p}, \tilde{q})$. i Spherical cylindrical projection diagrams $(\gamma, \zeta)$ and $(\tilde{\gamma}, \tilde{\delta})$.j Modified spherical cylindrical projection diagrams $(a, b)$ and $(\tilde{a}, \tilde{b}) \cdot \mathbf{k}$ Spherical cylindrical orthogonal projection diagrams $(\chi, \zeta)$ and $(\tilde{\chi}, \tilde{\zeta})$

details for this projection are provided in "Appendix 9." Under this projection, the mixing fraction of DC and CLVD in the deviatoric component with respect to scalar moment is orthogonal to the ratio of the isotropic component to the total. Thus, we can recognize by visual inspection when a deviatoric component lies close to DC or \pm CLVD, as well as how much of an isotropic component is included in a solution. The square shape also has an advantage in application in terms of usability. However, a completely isotropic source mechanism will be projected onto a line, which might be a disadvantage.

In some cases, it could be useful to modify the cylindrical projection to use a triangle shape, so that \pm ISO is projected onto points, rather than lines. We illustrate such a modification, with areal density kept constant, in 
Fig. 4j. This "modified spherical cylindrical projection" (j) is given by

$$
\left[\begin{array}{l}
a \\
b
\end{array}\right]=\left[\begin{array}{c}
-\frac{6}{\pi} \tan ^{-1}\left(\frac{\lambda_{1}-2 \lambda_{2}+\lambda_{3}}{\sqrt{3}\left(\lambda_{1}-\lambda_{3}\right)}\right) \sqrt{1-\frac{\left|\lambda_{1}+\lambda_{2}+\lambda_{3}\right|}{\sqrt{3\left(\lambda_{1}^{2}+\lambda_{2}^{2}+\lambda_{3}^{2}\right)}}} \\
\frac{\left(\lambda_{1}+\lambda_{2}+\lambda_{3}\right) / \sqrt{3\left(\lambda_{1}^{2}+\lambda_{2}^{2}+\lambda_{3}^{2}\right)}}{1+\sqrt{1-\left|\lambda_{1}+\lambda_{2}+\lambda_{3}\right| / \sqrt{3\left(\lambda_{1}^{2}+\lambda_{2}^{2}+\lambda_{3}^{2}\right)}}}
\end{array}\right] .
$$

The +CLVD coordinate correction here is $(\tilde{a}, \tilde{b})=(-a, b)$. Although this projection has not been reported in any previous study, this diagram is the only diamond-shaped projection that preserves both density and horizontal lines from a three-dimensional lune. Full details for this projection appear in "Appendix 10."

Orthogonal projection of a cylinder is also possible, although the result is not equal area (Fig. 4k). This idea was proposed by Zhu and Ben-Zion (2013), though they did not produce an illustrative diagram. This "spherical cylindrical orthogonal projection" ( $k$ ) is given by

$$
\left[\begin{array}{l}
\chi \\
\zeta
\end{array}\right]=\left[\begin{array}{c}
\frac{-\left(\lambda_{1}-2 \lambda_{2}+\lambda_{3}\right) / 2}{\sqrt{\lambda_{1}^{2}+\lambda_{2}^{2}+\lambda_{3}^{2}-\lambda_{1} \lambda_{2}-\lambda_{2} \lambda_{3}-\lambda_{1} \lambda_{3}}} \\
\frac{\lambda_{1}+\lambda_{2}+\lambda_{3}}{\sqrt{3\left(\lambda_{1}^{2}+\lambda_{2}^{2}+\lambda_{3}^{2}\right)}}
\end{array}\right] .
$$

Its details appear in "Appendix 11." This is similar to the spherical cylindrical projection, but the areal density is slightly inhomogeneous relative to the lune. To facilitate comparison, we use the coordinate normalization $(\tilde{\chi}, \tilde{\zeta})=(-2 \chi, \zeta)$

\section{Other projections}

Although we have tried to explain most of the diagrams using the three-dimensional eigenvalue space $\left(\lambda_{1}, \lambda_{2}, \lambda_{3}\right)$, some representations cannot be written as a one-to-one projection of this space.

Vavryčuk (2001) suggested two parameters to explain the mixing ratios of DC and CLVD and the ratio of ISO to the total; the variables $-\epsilon$ and $c^{\mathrm{ISO}}$ in the original paper are equivalent to $\epsilon$ and $v$ in the present study, respectively. The first parameter, $\epsilon$, is often used to describe the percentage of CLVD (e.g., Kuge and Lay 1994b). The second parameter is identical to the term $v$ introduced by Hudson et al. (1989). Using these parameters, we can produce the source diagram shown in Fig. 5l. We call this diagram "percentile projection" (l), since Vavryčuk (2001) introduced this parameterization to explain these percentiles. Although the previous study did not propose a two-dimensional projection based on this, we include such a projection because these parameters can be used to form a two-dimensional diagram straightforwardly. This projection ("Appendix 12" section) is given by

$$
\left[\begin{array}{c}
\epsilon \\
v
\end{array}\right]=\left[\begin{array}{c}
\frac{-2\left(\lambda_{1}-2 \lambda_{2}+\lambda_{3}\right)}{3\left(\lambda_{1}-\lambda_{3}\right)+\left|\lambda_{1}-2 \lambda_{2}+\lambda_{3}\right|} \\
\frac{\lambda_{1}+\lambda_{2}+\lambda_{3}}{3 \max \left(\lambda_{1},-\lambda_{3}\right)}
\end{array}\right] .
$$

Considering that the horizontal coordinate takes values between -0.5 and 0.5 , we use the normalized coordinates $(\tilde{\epsilon}, \tilde{v})=(-2 \epsilon, v)$ for comparison with other projections.

The percentile projection can be transformed into a diamond shape to represent the relative fractions of $\mathrm{DC}$, CLVD, and ISO more intuitively, by rescaling the horizontal position based on the vertical position (Fig. $5 \mathrm{~m}$ ). Such a parameterization was introduced by Vavryčuk (2001); $c$ and $v$ in the present study correspond to $-c$ CLVD and $c^{\mathrm{ISO}}$ in the original paper, respectively. This "modified percentile projection" $(\mathrm{m})$ is written

$\left[\begin{array}{c}c \\ v\end{array}\right]=\left[\begin{array}{c}\frac{-4\left(\lambda_{1}-2 \lambda_{2}+\lambda_{3}\right)}{3\left(\lambda_{1}-\lambda_{3}\right)+\left|\lambda_{1}-2 \lambda_{2}+\lambda_{3}\right|}\left(1-\frac{\left|\lambda_{1}+\lambda_{2}+\lambda_{3}\right|}{3 \max \left(\lambda_{1},-\lambda_{3}\right)}\right) \\ \frac{\lambda_{1}+\lambda_{2}+\lambda_{3}}{3 \max \left(\lambda_{1},-\lambda_{3}\right)}\end{array}\right]$.

For comparison with other projections, we use coordinate normalization $(\tilde{c}, \tilde{v})=(-c, v)$, equivalent to $\left(c^{\mathrm{CLVD}}, c^{\mathrm{ISO}}\right)$ of Vavryčuk (2001). Further details appear in "Appendix 13."

Chapman and Leaney (2012) also introduced a diagram based on the concept of potency. Although an equivalent source-type diagram can be produced by assuming a specific type of medium (e.g., Poissonian), we do not consider potency-based diagrams in this study. We note that all the source-type diagrams listed above can be modified to describe potency tensors by making appropriate assumptions about the medium.

\section{Comparisons of diagrams}

We have described 13 diagrams, including complete transformation formulas. Here, we briefly summarize these projections, along with their respective advantages and disadvantages. Some important properties of each projection are listed in Table 2. Although each diagram has both advantages and disadvantages, and there is no perfect projection, we suggest that some diagrams are better projections than others.

\section{Diagram geometry}

With respect to their outlines, most diagrams have a square or diamond shape when normalized coordinates are used (Figs. 2, 3, 4, 5). We regard square and diamond source-type diagrams as more useful than others because their outlines are easier to draw. Since the domains of these diagrams are well defined, they are suitable for random searches or grid searches over a two-dimensional parameter space. 


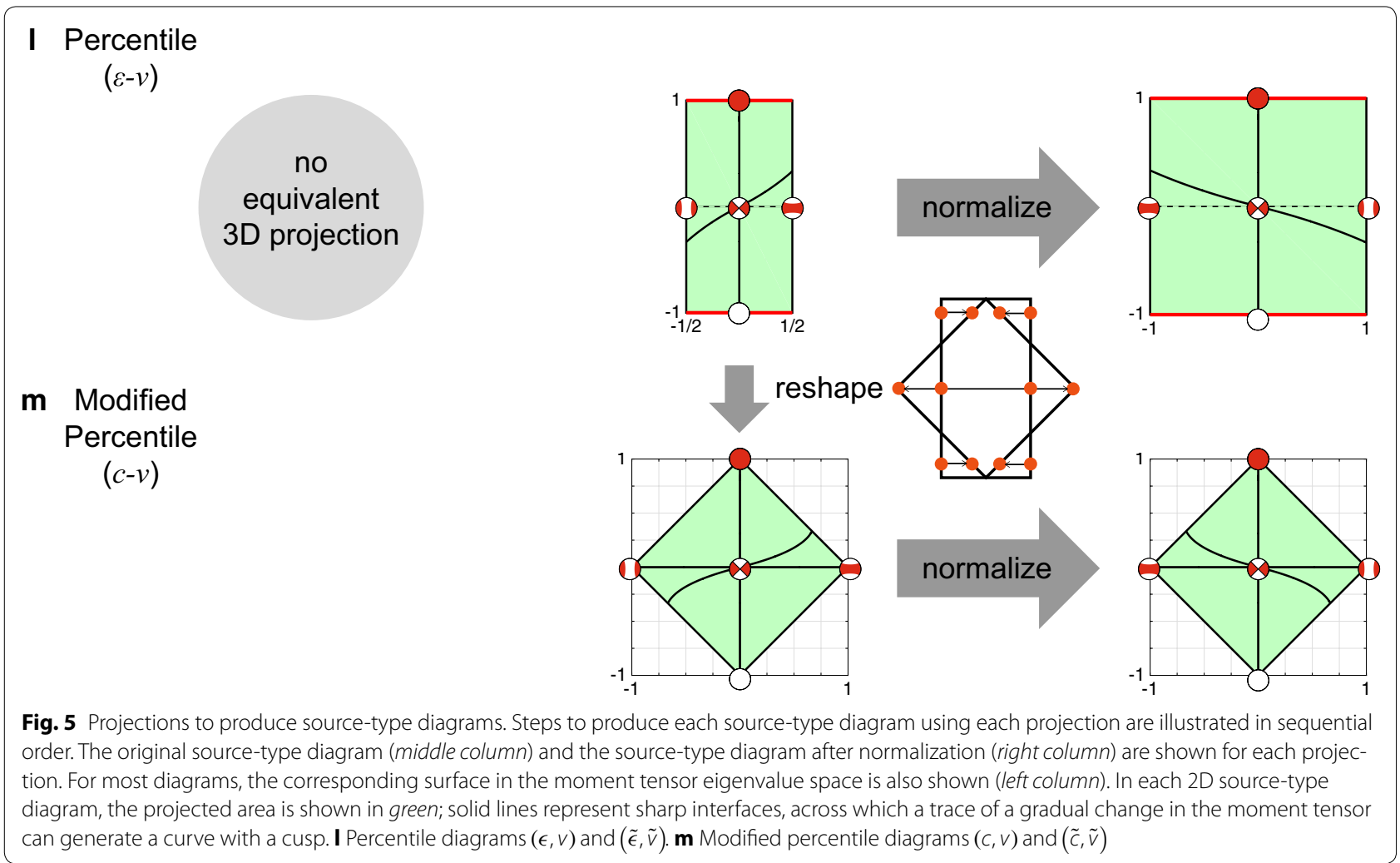

Table 2 Properties of source-type diagrams

\begin{tabular}{|c|c|c|c|c|c|}
\hline & & $\begin{array}{l}\text { Shape of normalized } \\
\text { diagram }\end{array}$ & $\begin{array}{l}\text { Sharp interface (except } \\
\text { inevitable one }{ }^{\text {a) }} \text { ) }\end{array}$ & $\begin{array}{l}\text { Laterally asymmetric } \\
\text { density }\end{array}$ & $\begin{array}{l}\text { Significant apparent den- } \\
\text { sity differences }\end{array}$ \\
\hline (a) & Cubic & Skewed rectangle & No (diagonal line) & Yes & No \\
\hline (b) & Hexagonal bi-pyramid & Diamond & $\begin{array}{l}\text { Vertical axis (horizontal } \\
\text { axis) }\end{array}$ & No & No \\
\hline (c) & $\begin{array}{l}\text { Modified hexagonal bi- } \\
\text { pyramid }\end{array}$ & Square & Vertical axis horizontal axis & No & Low density around $\pm I S O$ \\
\hline (d) & $\begin{array}{l}\text { Conjugate hexagonal bi- } \\
\text { pyramid }\end{array}$ & Diamond & No (horizontal axis) & No & No \\
\hline (e) & Spherical equirectangular & Square & No & No & Low density around \pm ISO \\
\hline (f) & Spherical orthogonal & Circle & No & No & Concentration around $\pm \mathrm{ISO}$ \\
\hline (g) & $\begin{array}{l}\text { Modified spherical } \\
\text { orthogonal }\end{array}$ & Diamond & No (horizontal axis) & No & $\begin{array}{l}\text { Concentration around r-s } \\
\text { axes }\end{array}$ \\
\hline (h) & Spherical azimuthal & Lemon shape & No & No & No \\
\hline (i) & Spherical cylindrical & Square & No & No & No \\
\hline (j) & $\begin{array}{l}\text { Modified spherical } \\
\text { cylindrical }\end{array}$ & Diamond & No (horizontal axis) & No & No \\
\hline (k) & $\begin{array}{l}\text { Spherical cylindrical } \\
\text { orthogonal }\end{array}$ & Square & No & No & No \\
\hline$(\mathrm{l})$ & Percentile & Square & Vertical axis diagonal line & Yes & Low density around $\pm 1 S O$ \\
\hline (m) & Modified percentile & Diamond & $\begin{array}{l}\text { Vertical axis diagonal line } \\
\text { (horizontal axis) }\end{array}$ & Yes & No \\
\hline
\end{tabular}

\footnotetext{
a The sharp interface that is inevitable because of the angular outline shape is enclosed in parentheses
} 
A square diagram is the easiest to produce, though not a one-to-one projection for \pm ISO mechanisms ( \pm ISO is projected onto lines). On the other hand, a diamond diagram is a one-to-one projection for all mechanisms, but the horizontal axis has a singularity. For example, combinations of + CLVD and +ISO in Fig. 2b trace the bending outline of the diagram, which contains a cusp. Although a gradual change in mechanism is mostly represented by a smooth polyline, the polyline develops a sharp cusp when the mechanism changes in such a way that its diagram representation crosses the horizontal axis. Since the combination of two different mechanisms, which is thought to exist in nature, could be visualized as a "non-smooth line," source-type distributions across the "sharp interface" have to be interpreted carefully. The diagonal line in the cubic projection (a) is also a sharp interface. In this study, we do not consider the sharp interface a disadvantage; rather, we claim such an edge is an inevitable consequence of the outline shape.

All sharp interfaces are shown as solid lines in twodimensional diagrams in Figs. 2, 3, 4 and 5. An additional sharp interface exists in some diagrams, which we consider a disadvantage. This can be found in the vertical axes of the hexagonal bi-pyramid projection (b) and its modified form (c), which are generated by connecting triangular surfaces in the three-dimensional eigenvalue space. As this "non-smooth" nature follows from an absolute value symbol in the formula, the percentile and modified percentile projections ( 1 and $\mathrm{m}$ ) also have this disadvantage.

\section{Apparent density differences}

Next, we discuss the apparent density differences between diagrams, which affect their relative visual interpretability. We first compare their relative appearances using real data. As an example of a global catalog, we use the Preliminary Determination of Epicenters (PDE) catalog, maintained by the United States Geological Survey (USGS), which combines data from multiple regional and global subcatalogs. We use normalized coordinates for each diagram to enable an effective comparison (Fig. 6). All data are plotted in the same color, regardless of subcatalog of origin. Some of the non-double-couple components may be artifacts, including isotropic components, but we can still use them in comparative diagrams. More than half of all events $(19,808$ out of 35,403$)$ have no isotropic component, which is thought to be partly because of the assumptions underlying their respective moment tensor solutions; these events are densely distributed on the horizontal axis, with only 310 DC events. The event distributions look similar on almost all diagrams, except for the modified spherical orthogonal projection (Fig. 6g), for which the projected points are concentrated near the center.

To compare the apparent density differences in greater detail, we create projections of synthetic data with a wider variety of source types in Fig. 7 (Additional files 1, 2). We generate eigenvalues for each synthetic mechanism using independent standard normal distributions. Although a plausible candidate for the true distribution of moment tensors was suggested by Tape and Tape (2015), the true distribution is not yet well known and may depend on tectonic setting, location, or analysis method; for this reason, we use a simplified distribution to generate reference data covering the full range of source mechanisms (i.e., between DC, CLVD, and ISO). Since these synthetic data are produced homogeneously on a spherical surface in three-dimensional eigenvalue space, the areal density is kept constant in the spherical azimuthal, spherical cylindrical, and modified spherical cylindrical projections $(\mathrm{h}-\mathrm{j})$. However, this does not imply that these diagrams are better than the others, as the synthetic distribution is an artificial reference; for example, if we instead created synthetic data, under the assumption that each eigenvalue had an independent uniform distribution, then the cubic projection (a) would become homogeneous. Here, we use synthetic data only to compare the relative density differences between diagrams. Although areal density can be formulated mathematically using the Jacobean, scatter plots of synthetic data provide enough information about apparent differences for the purposes of discussion.

A horizontally asymmetric distribution can be seen for the cubic projection (a) and the percentile and modified percentile projections ( $\mathrm{l}$ and $\mathrm{m}$ ). Although the deviatoric and isotropic components might depend on one another, as for a fixed Poisson's ratio, we do not know their exact relationship. Considering that the synthetic data were produced by assuming independent deviatoric and isotropic components, each diagram is expected to show a symmetric distribution. For this reason, we consider the asymmetry of these diagrams a disadvantage.

We now focus on the observed density concentrations near deviatoric mechanisms with the PDE catalog and the modified spherical orthogonal projection (g). The synthetic data in Fig. $7 \mathrm{~g}$ show that points are concentrated on both the horizontal axis and the vertical axis; this is understandable, because each coordinate value is squared from the original spherical orthogonal projection (f). We regard the concentration of points as a disadvantage, however, because significant differences between mechanisms can potentially be concealed by this diagram.

The areal densities in plot regions close to isotropic mechanisms are found to be low for the modified hexagonal bi-pyramid projection (c), spherical equirectangular projection (e), and percentile projection (l), in which even near-isotropic mechanisms may be projected apart from the isotropic mechanism. On the other hand, the spherical orthogonal projection (f) and modified spherical 
a

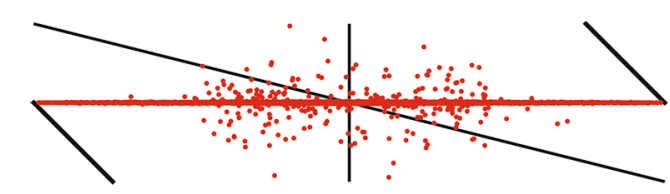

c

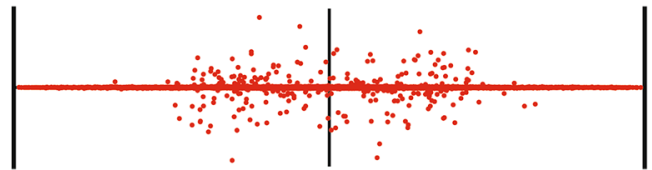

e

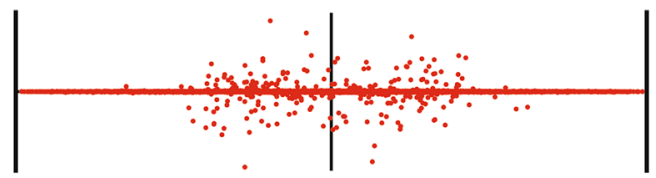

g

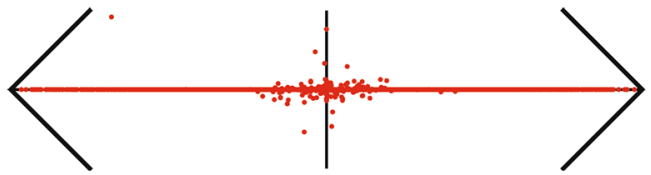

i

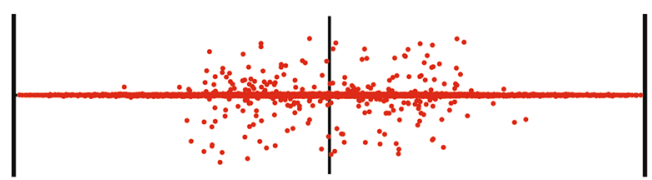

k

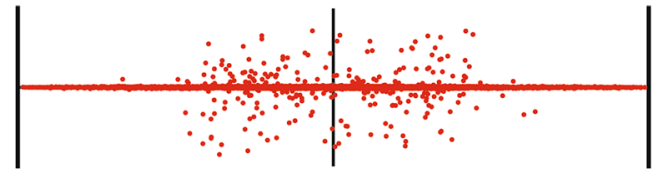

m

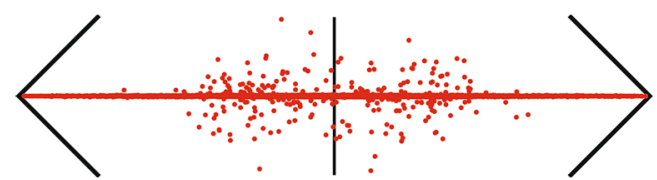

b

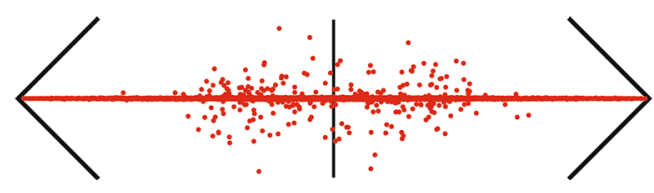

d

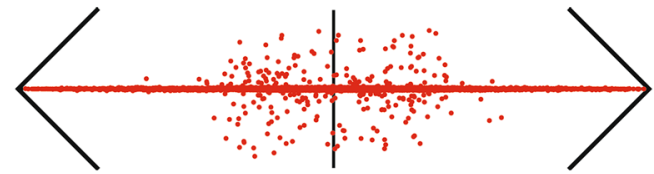

f

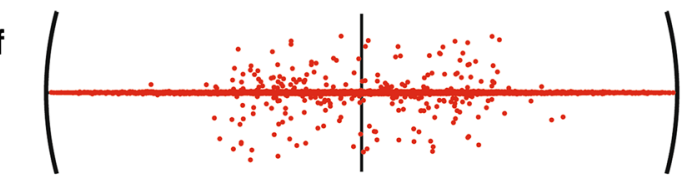

h

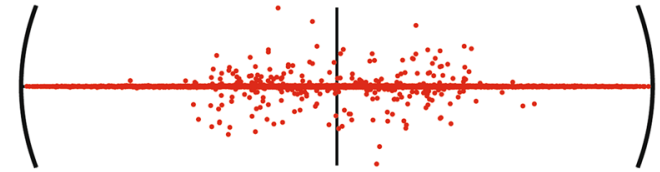

j

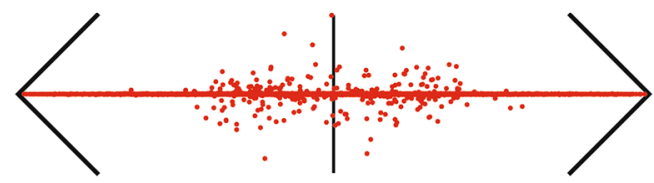

I

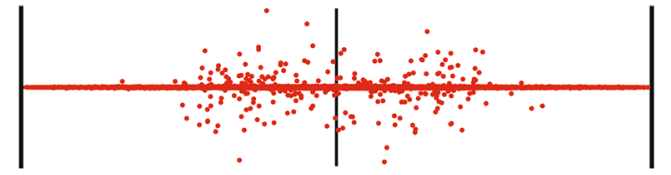

Fig. 6 PDE catalog projected onto source-type diagrams. Mechanisms for 35,403 events in the USGS Preliminary Determination of Epicenters (PDE) catalog, from February 1981 to July 2013, are shown after projection onto each source-type diagram. There are 19,808 events with deviatoric mechanisms, including 310 DC events. For these comparison plots, we use normalized diagrams, where DC, $\pm C L V D$, and $\pm I S O$ correspond to $(0,0)$ ,$(0, \pm 1)$, and $( \pm 1,0)$, respectively. Only parts of diagram with the horizontal range -1 to 1 and the vertical range -0.25 to 0.25 are shown. a Cubic projection diagram $(\tilde{u}, \tilde{v})$. b Hexagonal bi-pyramid projection diagram $(\tilde{\tau}, \tilde{k})$. c Modified hexagonal bi-pyramid projection diagram $(\tilde{T}, \tilde{k})$. d Conjugate hexagonal bi-pyramid projection diagram $(\tilde{\eta}, \tilde{\xi})$. e Spherical equirectangular projection diagram $(\tilde{\gamma}, \tilde{\delta})$. f Spherical orthogonal projection $\operatorname{diagram}(\tilde{R}, \tilde{\zeta})$. g Modified spherical orthogonal projection diagram $(\tilde{r}, \tilde{S})$. (h) Spherical azimuthal projection diagram $(\tilde{p}, \tilde{q})$. i Spherical cylindrical projection diagram $(\tilde{\gamma}, \tilde{\zeta})$. j Modified spherical cylindrical projection diagram $(\tilde{a}, \tilde{b})$. $\mathbf{k}$ Spherical cylindrical orthogonal projection diagram $(\tilde{\chi}, \tilde{\zeta})$. I Percentile diagram $(\tilde{\epsilon}, \tilde{v}) \cdot \mathbf{m}$ Modified percentile diagram $(\tilde{c}, \tilde{v})$

(See figure on next page.)

Fig. 7 Synthetic catalog projected onto source-type diagrams. A synthetic catalog of 10,000 events, which covers a wide range of source types, is projected onto each source-type diagram. Moment tensor eigenvalues are produced using independent standard normal distributions. Diagram normalization is identical to that in Fig. 6. The horizontal and vertical ranges are -1 to 1 , except for the cubic projection (a), for which the horizontal range is $-4 / 3$ to $4 / 3$. a Cubic projection diagram $(\tilde{u}, \tilde{v})$. b Hexagonal bi-pyramid projection diagram $(\tilde{\tau}, \tilde{k})$. c Modified hexagonal bi-pyramid projection diagram $(\tilde{T}, \tilde{k})$.d Conjugate hexagonal bi-pyramid projection diagram $(\tilde{\eta}, \tilde{\xi})$. e Spherical equirectangular projection diagram $(\tilde{\gamma}, \tilde{\delta})$. f Spherical orthogonal projection diagram $(\tilde{R}, \tilde{\zeta})$. $\mathbf{g}$ Modified spherical orthogonal projection diagram $(\tilde{r}, \tilde{s})$. h Spherical azimuthal projection diagram $(\tilde{p}, \tilde{q})$. i Spherical cylindrical projection diagram $(\tilde{\gamma}, \tilde{\zeta})$.j Modified spherical cylindrical projection diagram $(\tilde{a}, \tilde{b})$. k Spherical cylindrical orthogonal projection diagram $(\tilde{\chi}, \tilde{\zeta})$. I Percentile diagram $(\tilde{\epsilon}, \tilde{v})$. $\mathbf{m}$ Modified percentile diagram $(\tilde{c}, \tilde{v})$ 


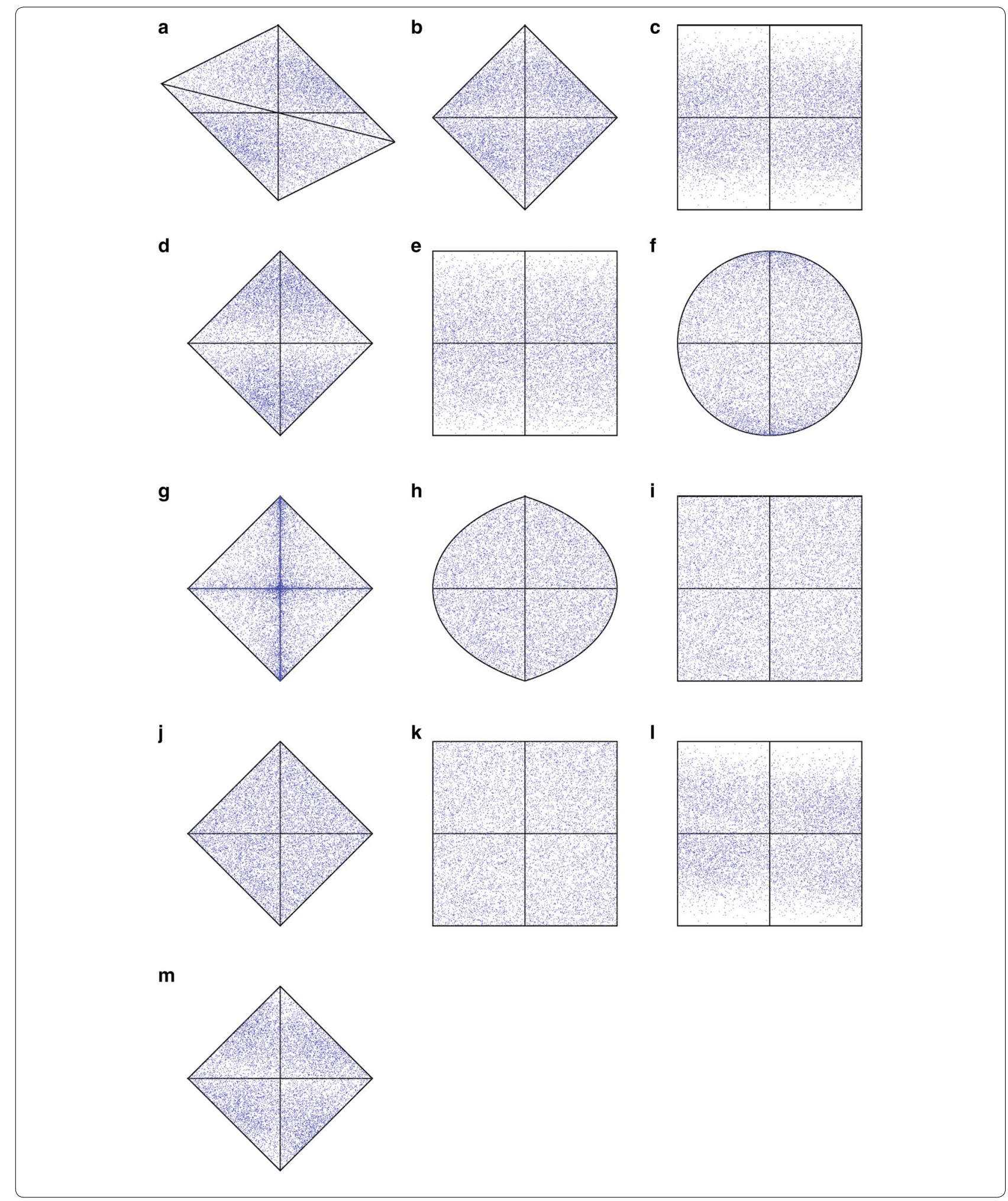

orthogonal projection (g) have concentrations near \pm ISO, which make them poorly suited to discuss variations in near-isotropic mechanisms.
Detailed distributions near DC, \pm CLVD, and \pm ISO are visualized using another synthetic catalog in Fig. 8. For each end-member mechanism, the eigenvalues are assumed to 
a

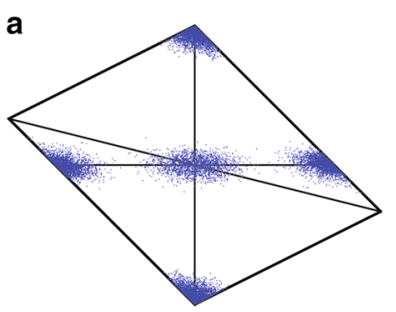

d

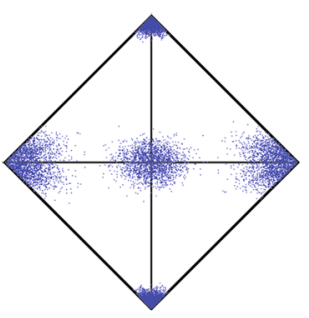

g

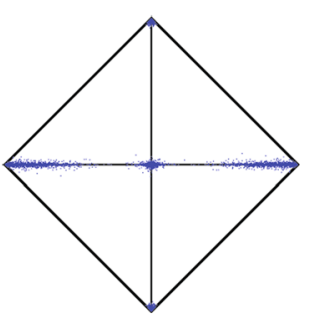

j

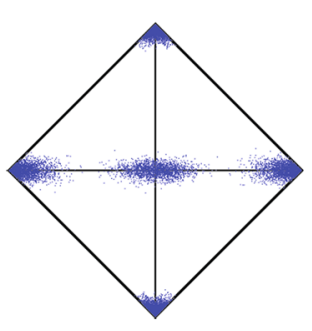

m

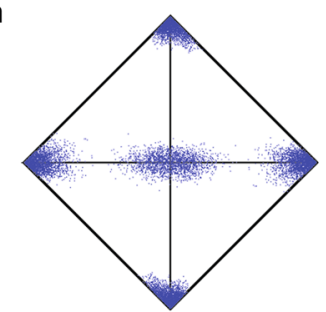

b

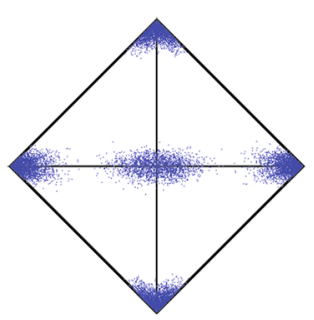

e

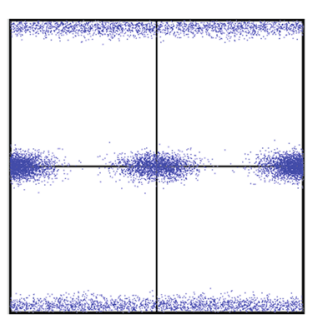

h

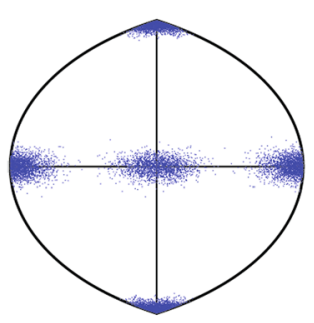

k

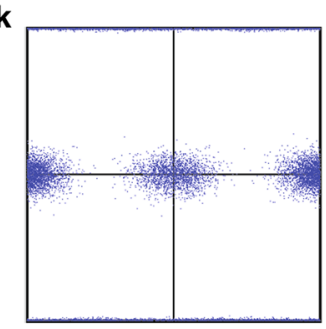

c

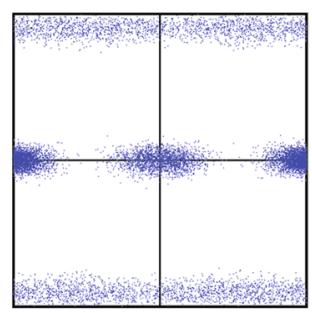

f

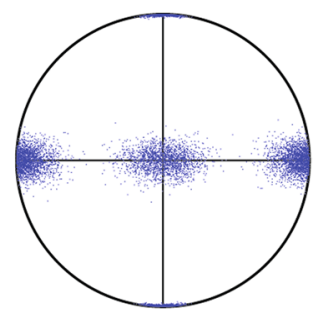

i

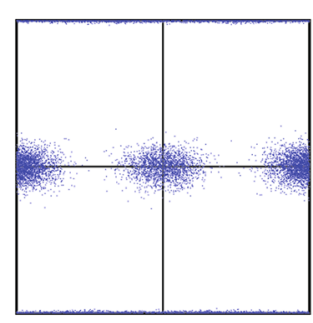

I

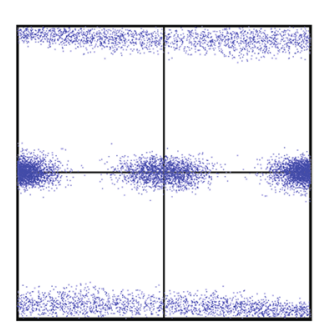

Fig. 8 End-member synthetic catalog projected onto source-type diagrams. A synthetic catalog produced near the end-member source mechanisms (DC, $\pm C L V D$, and $\pm I S O$ ) is projected onto each source-type diagram. For each mechanism, we produced 2000 events from eigenvalues that follow independent normal distributions with $\sigma=10 \%$ of the total scalar moment. Diagram normalization and axes ranges are identical to Fig. 7. a Cubic projection diagram $(\tilde{u}, \tilde{v})$. b Hexagonal bi-pyramid projection diagram $(\tilde{\tau}, \tilde{k})$. c Modified hexagonal bi-pyramid projection diagram $(\tilde{T}, \tilde{k})$. d Conjugate hexagonal bi-pyramid projection diagram $(\tilde{\eta}, \tilde{\xi})$. e Spherical equirectangular projection diagram $(\tilde{\gamma}, \tilde{\delta})$. f Spherical orthogonal projection diagram $(\tilde{R}, \tilde{\zeta})$. g Modified spherical orthogonal projection diagram $(\tilde{r}, \tilde{s})$. h Spherical azimuthal projection diagram $(\tilde{p}, \tilde{q})$. i Spherical cylindrical projection diagram $(\tilde{\gamma}, \tilde{\zeta})$. j Modified spherical cylindrical projection diagram $(\tilde{a}, \tilde{b})$. $\mathbf{k}$ Spherical cylindrical orthogonal projection diagram $(\tilde{\chi}, \tilde{\zeta})$. I Percentile diagram $(\tilde{\epsilon}, \tilde{v}) \cdot \mathbf{m}$ Modified percentile diagram $(\tilde{\boldsymbol{c}}, \tilde{v})$ 
follow independent normal distributions whose standard deviations are $10 \%$ of the total scalar moment; these distributions thus form a synthetic catalog of end-member mechanisms with uncertainties. From visual inspection of the figure, we can easily see the concentrations near the deviatoric mechanisms for (g), and the isotropic mechanisms for (f) and (g). We also note that near-isotropic mechanisms occur apart from strictly isotropic mechanisms in (c), (e), and (l). We consider these points to be disadvantages.

While the distribution in Fig. 8 corresponds to a simplified representation of uncertainties, the distribution of the estimation errors for real observations is given by the six-parameter covariance matrix. In addition to estimation errors from the linear inversion, there are other contributions that affect uncertainties: for example, use of incorrect velocity model or arrival time picking errors. These effects of model uncertainties can be evaluated by adding perturbations to the assumed velocity model or the picked arrival times. We note that all of these uncertainties can be visualized in the source-type diagram as scatter plots (e.g., Aso and Ide 2014), density contours, or the distribution of variance reductions (e.g., Nayak and Dreger 2015). The representations of solution uncertainties, as well as the solutions themselves, are important aspects of the diagram.

We noted in the introduction that source-type diagrams can be useful for inversion, because we sometimes need to constrain the source type itself (e.g., Ford et al. 2010; Nayak and Dreger 2015). Source-type diagrams are useful tools in selecting various source types. We note that the mechanisms in Fig. 7 around which high densities are observed tend to be sparsely sampled when we select points evenly in a source-type diagram.

\section{Preferred diagrams}

From the viewpoint of usability, we suggest using diagrams that do not have any significant disadvantages, although we hesitate to categorically rule out the others (Table 2). The spherical cylindrical diagram (i) and spherical cylindrical orthogonal projection $(\mathrm{k})$, which are almost the same, are more advantageous than other square diagrams. If the preference is a diamond-shaped diagram, analogous to the triangular diagrams used for other purposes such as faulting types (Frohlich 1992) or mineral phases, then the conjugate hexagonal bi-pyramid projection (d) or modified spherical cylindrical diagram (j) is recommended.

Even though there are no significant differences between these four diagrams, we feel that stating our preference may be helpful for future applications. Invoking Occam's razor, it might be better to use diagrams on which a homogeneous distribution corresponds to the simplest distribution in the eigenvalue space, namely a homogeneous distribution on a surface of constant scalar moment. Diamond-shaped diagrams, on which all the end-member mechanisms of
DC, \pm CLVD, and \pm ISO are represented by points, can be understood more intuitively in a variety of research fields. As a result, the modified spherical cylindrical diagram (j) is the preferred diagram for a variety of applications in seismology, volcanology, geology, and tectonics.

\section{Conclusions}

We reviewed 13 representations of seismic source types, with complete mathematical formulas, and evaluated differences between them. There is no single perfect projection of source type, and there could be an infinite number of possible projections, as with map projections. It is valuable to categorize projections and to recognize the differences between them. We need to pay particular attention to the apparent density difference. Based on geometry and density distributions of synthetic and real data, we favor the use of a conjugate hexagonal projection (d), spherical cylindrical projection (i), modified spherical cylindrical projection (j), or spherical cylindrical orthogonal projection $(\mathrm{k})$. Of these choices, the modified spherical cylindrical diagram ( $j$ ) is the easiest projection to use. Finally, we again point out that we must always consider the apparent differences between diagrams when deciding which source-type diagram is most appropriate to use.

\section{Additional files}

Additional file 1. MATLAB function for conversion between moment tensor eigenvalues and coordinates in source-type diagrams. An example to use this function is provided as Additional file 2.

Additional file 2. MATLAB program to project random points in the moment tensor eigenvalue space into source-type diagrams. This source code requires the function of Additional file 1 .

\section{Abbreviations}

DC: double-couple; CLVD: compensated linear vector dipole; ISO: isotropic deformation.

\section{Authors' contributions}

NA derived the equations, made the figures, and wrote the manuscript. KO contributed to the derivations. SI supervised this study as an advisor and provided perspectives of importance to this study. All authors read and approved the final manuscript.

\section{Authors' information}

This study was initiated when NA and KO were PhD students and SI was their advisor at the University of Tokyo.

\section{Author details}

1 Seismological Laboratory, California Institute of Technology, 1200 E. Calif. Blvd., Pasadena, CA 91125, USA. ${ }^{2}$ Disaster Prevention Research Institute, Kyoto University, Gokasho, Uji, Kyoto 611-0011, Japan. ${ }^{3}$ School of Science, The University of Tokyo, 7-3-1 Hongo, Bunkyo, Tokyo 113-0033, Japan.

\section{Acknowledgements}

We used the moment tensor data from the Preliminary Determination of Epicenters (PDE) catalog, distributed by the United States Geological Survey (USGS). This work was supported by JSPS (Research Project Number: 12J09135). We thank Daniel C. Bowden for useful discussions. We also thank Václav Vavryčuk and Douglas S. Dreger for elaborative and constructive reviews and 
comments. MATLAB code for conversion between moment tensor eigenvalues and coordinates in source-type diagrams is provided as Additional files.

\section{Competing interests}

The authors declare that they have no competing interests.

\section{Appendix 1: (a) Cubic projection ( $u-v)$}

For $\lambda_{1} \geq-\lambda_{3}$, the $u-v$ coordinate system projects DC $\left(\lambda_{1}, \lambda_{2}, \lambda_{3}\right)=(1,0,-1)$ onto $(u, v)=(0,0)$, +CLVD $\left(\lambda_{1}, \lambda_{2}, \lambda_{3}\right)=(1,-1 / 2,-1 / 2)$ onto $(u, v)=(-1,0)$, and + ISO $\left(\lambda_{1}, \lambda_{2}, \lambda_{3}\right)=(1,1,1)$ onto $(u, v)=(0,1)$. Such a linear function is given by

$$
\left[\begin{array}{l}
\lambda_{1} \\
\lambda_{2} \\
\lambda_{3}
\end{array}\right]=A_{c}\left[\begin{array}{ccc}
1 & 1 & 1 \\
0 & -1 / 2 & 1 \\
-1 & -1 / 2 & 1
\end{array}\right]\left[\begin{array}{c}
1+u-v \\
-u \\
v
\end{array}\right] .
$$

Solving this linear problem, we find

$$
\left[\begin{array}{l}
1 \\
u \\
v
\end{array}\right]=\left[\begin{array}{c}
\lambda_{1} / A_{c} \\
\frac{-2\left(\lambda_{1}-2 \lambda_{2}+\lambda_{3}\right)}{3 A_{c}} \\
\frac{\lambda_{1}+\lambda_{2}+\lambda_{3}}{3 A_{c}}
\end{array}\right]
$$

For $\lambda_{1} \leq-\lambda_{3}$, the $u-v$ coordinate system projects DC $\left(\lambda_{1}, \lambda_{2}, \lambda_{3}\right)=(1,0,-1)$ onto $(u, v)=(0,0),-$ CLVD $\left(\lambda_{1}, \lambda_{2}, \lambda_{3}\right)=(1 / 2,1 / 2,-1)$ onto $(u, v)=(1,0)$, and ISO $\left(\lambda_{1}, \lambda_{2}, \lambda_{3}\right)=(-1,-1,-1)$ onto $(u, v)=(0,-1)$, which is given by

$$
\left[\begin{array}{l}
\lambda_{1} \\
\lambda_{2} \\
\lambda_{3}
\end{array}\right]=A_{c}\left[\begin{array}{ccc}
1 & 1 / 2 & -1 \\
0 & 1 / 2 & -1 \\
-1 & -1 & -1
\end{array}\right]\left[\begin{array}{c}
1-u+v \\
u \\
-v
\end{array}\right] .
$$

Solving this linear problem, we find

$$
\left[\begin{array}{l}
1 \\
u \\
v
\end{array}\right]=\left[\begin{array}{c}
-\lambda_{3} / A_{c} \\
\frac{-2\left(\lambda_{1}-2 \lambda_{2}+\lambda_{3}\right)}{3 A_{c}} \\
\frac{\lambda_{1}+\lambda_{2}+\lambda_{3}}{3 A_{c}}
\end{array}\right]
$$

Combining these two cases (16) and (18), we obtain

$$
\left[\begin{array}{c}
A_{c} \\
u \\
v
\end{array}\right]=\left[\begin{array}{c}
\max \left(\lambda_{1},-\lambda_{3}\right) \\
\frac{-2\left(\lambda_{1}-2 \lambda_{2}+\lambda_{3}\right)}{3 \max \left(\lambda_{1},-\lambda_{3}\right)} \\
\frac{\lambda_{1}+\lambda_{2}+\lambda_{3}}{3 \max \left(\lambda_{1},-\lambda_{3}\right)}
\end{array}\right]
$$

The normalized coordinate system in which \pm CLVD corresponds to $(\tilde{u}, \tilde{v})=( \pm 1,0)$ is $(\tilde{u}, \tilde{v})=(-u, v)$.

Considering $\lambda_{1} \lesseqgtr-\lambda_{3} \Leftrightarrow u-4 v \gtreqless 0$, the combination of (15) and (17) results in the inverse transformation

$$
\left[\begin{array}{l}
\lambda_{1} \\
\lambda_{2} \\
\lambda_{3}
\end{array}\right]=\frac{A_{c}}{2}\left[\begin{array}{c}
\min (4 v-u, 0)+2 \\
2 v+u \\
\max (4 v-u, 0)-2
\end{array}\right] .
$$

\section{Appendix 2: (b) Hexagonal bi-pyramid projection}

\section{$(\tau-k)$}

For $\lambda_{1}-2 \lambda_{2}+\lambda_{3} \geq 0$, the $\tau-k$ coordinate system projects $\left(\lambda_{1}, \lambda_{2}, \lambda_{3}\right)=(1,0,-1)$ onto $(\tau, k)=(0,0),+$ CLVD $\left(\lambda_{1}, \lambda_{2}, \lambda_{3}\right)=(1,-1 / 2,-1 / 2)$ onto $(\tau, k)=(-1,0)$, and $\pm \mathrm{ISO}\left(\lambda_{1}, \lambda_{2}, \lambda_{3}\right)=( \pm 1, \pm 1, \pm 1)$ onto $(\tau, k)=(0, \pm 1)$, which is given by

$$
\left[\begin{array}{l}
\lambda_{1} \\
\lambda_{2} \\
\lambda_{3}
\end{array}\right]=A_{h 1}\left[\begin{array}{ccc}
1 & 1 & 1 \\
0 & -1 / 2 & 1 \\
-1 & -1 / 2 & 1
\end{array}\right]\left[\begin{array}{c}
1+\tau-|k| \\
-\tau \\
k
\end{array}\right] .
$$

This linear problem can be solved as

$$
\left[\begin{array}{c}
1-|k| \\
\tau \\
k
\end{array}\right]=\frac{1}{3 A_{h 1}}\left[\begin{array}{ccc}
2 & -1 & -1 \\
-2 & 4 & -2 \\
1 & 1 & 1
\end{array}\right]\left[\begin{array}{l}
\lambda_{1} \\
\lambda_{2} \\
\lambda_{3}
\end{array}\right] .
$$

Accordingly,

$$
\left[\begin{array}{c}
A_{h 1} \\
\tau \\
k
\end{array}\right]=\left[\begin{array}{c}
\frac{2 \lambda_{1}-\lambda_{2}-\lambda_{3}+\left|\lambda_{1}+\lambda_{2}+\lambda_{3}\right|}{3} \\
\frac{-2\left(\lambda_{1}-2 \lambda_{2}+\lambda_{3}\right)}{3 A_{h 1}} \\
\frac{\lambda_{1}+\lambda_{2}+\lambda_{3}}{3 A_{h 1}}
\end{array}\right] .
$$

For $\lambda_{1}-2 \lambda_{2}+\lambda_{3} \leq 0$, the $\tau-k$ coordinate system projects DC $\left(\lambda_{1}, \lambda_{2}, \lambda_{3}\right)=(1,0,-1) \quad$ onto $(\tau, k)=(0,0),-\operatorname{CLVD}\left(\lambda_{1}, \lambda_{2}, \lambda_{3}\right)=(1 / 2,1 / 2,-1)$ onto $(\tau, k)=(1,0)$, and $\pm \operatorname{ISO}\left(\lambda_{1}, \lambda_{2}, \lambda_{3}\right)=( \pm 1, \pm 1, \pm 1)$ onto $(\tau, k)=(0, \pm 1)$, which is given by

$$
\left[\begin{array}{l}
\lambda_{1} \\
\lambda_{2} \\
\lambda_{3}
\end{array}\right]=A_{h 1}\left[\begin{array}{ccc}
1 & 1 / 2 & 1 \\
0 & 1 / 2 & 1 \\
-1 & -1 & 1
\end{array}\right]\left[\begin{array}{c}
1-\tau-|k| \\
\tau \\
k
\end{array}\right] .
$$

We thus get

$$
\left[\begin{array}{c}
1-|k| \\
\tau \\
k
\end{array}\right]=\frac{1}{3 A_{h 1}}\left[\begin{array}{ccc}
1 & 1 & -2 \\
-2 & 4 & -2 \\
1 & 1 & 1
\end{array}\right]\left[\begin{array}{l}
\lambda_{1} \\
\lambda_{2} \\
\lambda_{3}
\end{array}\right],
$$

hence

$$
\left[\begin{array}{c}
A_{h 1} \\
\tau \\
k
\end{array}\right]=\left[\begin{array}{c}
\frac{\lambda_{1}+\lambda_{2}-2 \lambda_{3}+\left|\lambda_{1}+\lambda_{2}+\lambda_{3}\right|}{3} \\
\frac{-2\left(\lambda_{1}-2 \lambda_{2}+\lambda_{3}\right)}{3 A_{h 1}} \\
\frac{\lambda_{1}+\lambda_{2}+\lambda_{3}}{3 A_{h 1}}
\end{array}\right] .
$$

Both (23) and (26) satisfy

$$
\left[\begin{array}{c}
A_{h 1} \\
\tau \\
k
\end{array}\right]=\left[\begin{array}{c}
\frac{3\left(\lambda_{1}-\lambda_{3}\right)+\left|\lambda_{1}-2 \lambda_{2}+\lambda_{3}\right|+2\left|\lambda_{1}+\lambda_{2}+\lambda_{3}\right|}{6} \\
\frac{-4\left(\lambda_{1}-2 \lambda_{2}+\lambda_{3}\right)}{3\left(\lambda_{1}-\lambda_{3}\right)+\left|\lambda_{1}-2 \lambda_{2}+\lambda_{3}\right|+2\left|\lambda_{1}+\lambda_{2}+\lambda_{3}\right|} \\
\frac{2\left(\lambda_{1}+\lambda_{2}+\lambda_{3}\right)}{3\left(\lambda_{1}-\lambda_{3}\right)+\left|\lambda_{1}-2 \lambda_{2}+\lambda_{3}\right|+2\left|\lambda_{1}+\lambda_{2}+\lambda_{3}\right|}
\end{array}\right] .
$$

We introduce the normalized coordinate $\operatorname{system}(\tilde{\tau}, \tilde{k})=$ $(-\tau, k)$ so that \pm CLVD corresponds to $(\tilde{\tau}, \tilde{k})=( \pm 1,0)$. 
Considering $\lambda_{1}-2 \lambda_{2}+\lambda_{3} \gtreqless 0 \Leftrightarrow \tau \lesseqgtr 0$, the combination of (21) and (24) results in the inverse transformation

$$
\left[\begin{array}{l}
\lambda_{1} \\
\lambda_{2} \\
\lambda_{3}
\end{array}\right]=\frac{A_{h 1}}{2}\left[\begin{array}{c}
\min (4 k, 0)-\max (\tau, 0)+2 \\
2 k+\tau \\
\max (4 k, 0)-\min (\tau, 0)-2
\end{array}\right] .
$$

Appendix 3: (c) Modified hexagonal bi-pyramid projection $(T-k)$

Replacing (27) by $T=\tau /(1-|k|)$,

$$
\left[\begin{array}{c}
A_{h 1} \\
T \\
k
\end{array}\right]=\left[\begin{array}{c}
\frac{3\left(\lambda_{1}-\lambda_{3}\right)+\left|\lambda_{1}-2 \lambda_{2}+\lambda_{3}\right|+2\left|\lambda_{1}+\lambda_{2}+\lambda_{3}\right|}{6} \\
\frac{-4\left(\lambda_{1}-2 \lambda_{2}+\lambda_{3}\right)}{3\left(\lambda_{1}-\lambda_{3}\right)+\left|\lambda_{1}-2 \lambda_{2}+\lambda_{3}\right|} \\
\frac{2\left(\lambda_{1}+\lambda_{2}+\lambda_{3}\right)}{3\left(\lambda_{1}-\lambda_{3}\right)+\left|\lambda_{1}-2 \lambda_{2}+\lambda_{3}\right|+2\left|\lambda_{1}+\lambda_{2}+\lambda_{3}\right|}
\end{array}\right] .
$$

The normalized coordinate system $(\tilde{T}, \tilde{k})=(-T, k)$ projects \pm CLVD onto $(\tilde{T}, \tilde{k})=( \pm 1,0)$.

Replacing (28) by the inverse relationship $\tau=T(1-|k|)$,

$\left[\begin{array}{l}\lambda_{1} \\ \lambda_{2} \\ \lambda_{3}\end{array}\right]=\frac{A_{h 1}}{2}\left[\begin{array}{c}\min (4 k, 0)-(1-|k|) \max (T, 0)+2 \\ 2 k+(1-|k|) T \\ \max (4 k, 0)-(1-|k|) \min (T, 0)-2\end{array}\right]$.

\section{Appendix 4: (d) Conjugate hexagonal bi-pyramid} projection $(\eta-\zeta)$

The $\eta-\xi$ coordinate system projects DC $\left(\lambda_{1}, \lambda_{2}, \lambda_{3}\right)=(1,0,-1) \quad$ onto $\quad(\eta, \xi)=(0,0), \quad+$ CLVD $\left(\lambda_{1}, \lambda_{2}, \lambda_{3}\right)=(1,-1 / 2,-1 / 2)$ onto $(\eta, \xi)=(-1,0)$, and $\pm \operatorname{ISO}\left(\lambda_{1}, \lambda_{2}, \lambda_{3}\right)=( \pm 1, \pm 1, \pm 1)$ onto $(\eta, \xi)=(0, \pm 1)$, which is written

$$
\left[\begin{array}{l}
\lambda_{1} \\
\lambda_{2} \\
\lambda_{3}
\end{array}\right]=A_{h 2}\left[\begin{array}{ccc}
1 & 4 / 3 & 2 / 3 \\
0 & -2 / 3 & 2 / 3 \\
-1 & -2 / 3 & 2 / 3
\end{array}\right]\left[\begin{array}{c}
1+\eta-|\xi| \\
-\eta \\
\xi
\end{array}\right] .
$$

Solving this linear problem, we obtain

$$
\left[\begin{array}{c}
1-|\xi| \\
\eta \\
\xi
\end{array}\right]=\frac{1}{2 A_{h 2}}\left[\begin{array}{ccc}
1 & 0 & -1 \\
-1 & 2 & -1 \\
1 & 1 & 1
\end{array}\right]\left[\begin{array}{l}
\lambda_{1} \\
\lambda_{2} \\
\lambda_{3}
\end{array}\right]
$$

thus

$$
\left[\begin{array}{c}
A_{h 2} \\
\eta \\
\xi
\end{array}\right]=\left[\begin{array}{c}
\frac{\lambda_{1}-\lambda_{3}+\left|\lambda_{1}+\lambda_{2}+\lambda_{3}\right|}{2} \\
\frac{-\left(\lambda_{1}-2 \lambda_{2}+\lambda_{3}\right)}{\lambda_{1}-\lambda_{3}+\left|\lambda_{1}+\lambda_{2}+\lambda_{3}\right|} \\
\frac{\lambda_{1}+\lambda_{2}+\lambda_{3}}{\lambda_{1}-\lambda_{3}+\left|\lambda_{1}+\lambda_{2}+\lambda_{3}\right|}
\end{array}\right]
$$

The normalized coordinate system in which \pm CLVD corresponds to $(\tilde{\eta}, \tilde{\xi})=( \pm 1,0)$ is $(\tilde{\eta}, \tilde{\xi})=(-\eta, \xi)$.

Now (31) becomes the inverse transformation

$$
\left[\begin{array}{c}
\lambda_{1} \\
\lambda_{2} \\
\lambda_{3}
\end{array}\right]=\frac{A_{h 2}}{3}\left[\begin{array}{c}
2 \xi-\eta+3(1-|\xi|) \\
2 \xi+2 \eta \\
2 \xi-\eta-3(1-|\xi|)
\end{array}\right] .
$$

\section{Appendix 5: (e) Spherical equirectangular projection $(\gamma-\delta)$}

The norm for the spherical projection can be written as

$$
A_{s}=\sqrt{\lambda_{1}^{2}+\lambda_{2}^{2}+\lambda_{3}^{2}}=\sqrt{2} M_{o}
$$

and the projected point on the sphere is written as

$$
\left[\begin{array}{c}
x_{\text {lune }} \\
y_{\text {lune }} \\
z_{\text {lune }}
\end{array}\right]=\frac{1}{A_{s}}\left[\begin{array}{c}
\left(\lambda_{1}-\lambda_{3}\right) / \sqrt{2} \\
-\left(\lambda_{1}-2 \lambda_{2}+\lambda_{3}\right) / \sqrt{6} \\
\left(\lambda_{1}+\lambda_{2}+\lambda_{3}\right) / \sqrt{3}
\end{array}\right]=\left[\begin{array}{c}
\frac{\lambda_{1}-\lambda_{3}}{\sqrt{2\left(\lambda_{1}^{2}+\lambda_{2}^{2}+\lambda_{3}^{2}\right)}} \\
\frac{-\left(\lambda_{1}-\lambda_{2}+\lambda_{3}\right)}{\sqrt{6\left(\lambda_{1}^{2}+\lambda_{2}^{2}+\lambda_{3}^{2}\right)}} \\
\frac{\lambda_{1}+\lambda_{2}+\lambda_{3}}{\sqrt{3\left(\lambda_{1}^{2}+\lambda_{2}^{2}+\lambda_{3}^{2}\right)}}
\end{array}\right]
$$

in the Cartesian coordinates composed by $\mathbb{X}:\left(\lambda_{1}, \lambda_{2}, \lambda_{3}\right)=(1,0,-1) / \sqrt{2}$, $\mathbb{Y}:\left(\lambda_{1}, \lambda_{2}, \lambda_{3}\right)=(1,-2,1) / \sqrt{6}, \quad$ and $\mathbb{Z}:\left(\lambda_{1}, \lambda_{2}, \lambda_{3}\right)=(1,1,1) / \sqrt{3}$. Then, the longitude and latitude of the point are given by

$$
\left[\begin{array}{l}
\gamma \\
\delta
\end{array}\right]=\left[\begin{array}{c}
\tan ^{-1}\left(y_{\text {lune }} / x_{\text {lune }}\right) \\
\sin ^{-1} z_{\text {lune }}
\end{array}\right]=\left[\begin{array}{c}
-\tan ^{-1}\left(\frac{\lambda_{1}-2 \lambda_{2}+\lambda_{3}}{\sqrt{3}\left(\lambda_{1}-\lambda_{3}\right)}\right) \\
\sin ^{-1}\left(\frac{\lambda_{1}+\lambda_{2}+\lambda_{3}}{\sqrt{3\left(\lambda_{1}^{2}+\lambda_{2}^{2}+\lambda_{3}^{2}\right)}}\right)
\end{array}\right] .
$$

The normalized coordinate system $(\tilde{\gamma}, \tilde{\delta})=(-6 \gamma / \pi, 2 \delta / \pi)$ projects \pm CLVD at $(\tilde{\gamma}, \tilde{\delta})=( \pm 1,0)$ and \pm ISO at $(\tilde{\gamma}, \tilde{\delta})=(0, \pm 1)$.

Conversely, the point on the sphere is given by

$$
\left[\begin{array}{l}
x_{\text {lune }} \\
y_{\text {lune }} \\
z_{\text {lune }}
\end{array}\right]=\left[\begin{array}{c}
\cos \gamma \cos \delta \\
\sin \gamma \cos \delta \\
\sin \delta
\end{array}\right] \text {. }
$$

Given that this point in the original coordinates is

$$
\begin{aligned}
\frac{1}{\sqrt{2} M_{o}}\left[\begin{array}{l}
\lambda_{1} \\
\lambda_{2} \\
\lambda_{3}
\end{array}\right] & =\frac{1}{A_{s}}\left[\begin{array}{l}
\lambda_{1} \\
\lambda_{2} \\
\lambda_{3}
\end{array}\right] \\
& =\frac{x_{\text {lune }}}{\sqrt{2}}\left[\begin{array}{c}
1 \\
0 \\
-1
\end{array}\right]+\frac{y_{\text {lune }}}{\sqrt{6}}\left[\begin{array}{c}
-1 \\
2 \\
-1
\end{array}\right]+\frac{z_{\text {lune }}}{\sqrt{3}}\left[\begin{array}{l}
1 \\
1 \\
1
\end{array}\right],
\end{aligned}
$$

we get

$$
\left[\begin{array}{l}
\lambda_{1} \\
\lambda_{2} \\
\lambda_{3}
\end{array}\right]=\sqrt{2 / 3} M_{o}\left[\begin{array}{c}
\sin \delta-\sqrt{2} \sin \left(\gamma-\frac{\pi}{3}\right) \cos \delta \\
\sin \delta+\sqrt{2} \sin \gamma \cos \delta \\
\sin \delta-\sqrt{2} \sin \left(\gamma+\frac{\pi}{3}\right) \cos \delta
\end{array}\right] .
$$

\section{Appendix 6: (f) Spherical orthogonal projection $(R-\zeta)$}

The orthogonal projection of the lune is simply given by 


$$
\left[\begin{array}{c}
R \\
\zeta
\end{array}\right]=\left[\begin{array}{c}
y_{\text {lune }} \\
z_{\text {lune }}
\end{array}\right]=\left[\begin{array}{c}
\frac{-\left(\lambda_{1}-2 \lambda_{2}+\lambda_{3}\right)}{\sqrt{6\left(\lambda_{1}^{2}+\lambda_{2}^{2}+\lambda_{3}^{2}\right)}} \\
\frac{\lambda_{1}+\lambda_{2}+\lambda_{3}}{\sqrt{3\left(\lambda_{1}^{2}+\lambda_{2}^{2}+\lambda_{3}^{2}\right)}}
\end{array}\right]
$$

The normalized coordinate system in which \pm CLVD corresponds to $(\tilde{R}, \tilde{\zeta})=( \pm 1,0)$ is $(\tilde{R}, \tilde{\zeta})=(-2 R, \zeta)$.

Conversely, the point on the lune is given by

$$
\left[\begin{array}{c}
x_{\text {lune }} \\
y_{\text {lune }} \\
z_{\text {lune }}
\end{array}\right]=\left[\begin{array}{c}
\sqrt{1-R^{2}-\zeta^{2}} \\
R \\
\zeta
\end{array}\right]
$$

Substituting this equation into (39), we get the original coordinates as

$$
\left[\begin{array}{l}
\lambda_{1} \\
\lambda_{2} \\
\lambda_{3}
\end{array}\right]=\frac{M_{o}}{\sqrt{3}}\left[\begin{array}{c}
\sqrt{2} \zeta-R+\sqrt{3\left(1-R^{2}-\zeta^{2}\right)} \\
\sqrt{2} \zeta+2 R \\
\sqrt{2} \zeta-R-\sqrt{3\left(1-R^{2}-\zeta^{2}\right)}
\end{array}\right]
$$

\section{Appendix 7: (g) Modified spherical orthogonal projection $(r-s)$}

Taking square of each coordinate in the previous projection given by (41), we obtain a diamond-shaped diagram

$$
\left[\begin{array}{l}
r \\
s
\end{array}\right]=\left[\begin{array}{c}
R|R| \\
\zeta|\zeta|
\end{array}\right]=\left[\begin{array}{c}
\frac{-\left(\lambda_{1}-2 \lambda_{2}+\lambda_{3}\right)\left|\lambda_{1}-2 \lambda_{2}+\lambda_{3}\right|}{6\left(\lambda_{1}^{2}+\lambda_{2}^{2}+\lambda_{3}^{2}\right)} \\
\frac{\left(\lambda_{1}+\lambda_{2}+\lambda_{3}\right)\left|\lambda_{1}+\lambda_{2}+\lambda_{3}\right|}{3\left(\lambda_{1}^{2}+\lambda_{2}^{2}+\lambda_{3}^{2}\right)}
\end{array}\right] .
$$

The normalized coordinate system $(\tilde{r}, \tilde{s})=(-4 r, s)$ projects \pm CLVD at $(\tilde{r}, \tilde{s})=( \pm 1,0)$.

Using the relationship

$$
\left[\begin{array}{l}
R \\
\zeta
\end{array}\right]=\left[\begin{array}{l}
\operatorname{sgn}(r) \sqrt{|r|} \\
\operatorname{sgn}(s) \sqrt{|s|}
\end{array}\right],
$$

where $\operatorname{sgn}(x)$ represents a sign function, (43) can be replaced as

$$
\left[\begin{array}{l}
\lambda_{1} \\
\lambda_{2} \\
\lambda_{3}
\end{array}\right]=\frac{M_{o}}{\sqrt{3}}\left[\begin{array}{c}
\operatorname{sgn}(s) \sqrt{2|s|}-\operatorname{sgn}(r) \sqrt{|r|}+\sqrt{3(1-|r|-|s|)} \\
\operatorname{sgn}(s) \sqrt{2|s|}+2 \operatorname{sgn}(r) \sqrt{|r|} \\
\operatorname{sgn}(s) \sqrt{2|s|}-\operatorname{sgn}(r) \sqrt{|r|}-\sqrt{3(1-|r|-|s|)}
\end{array}\right] .
$$

\section{Appendix 8: (h) Spherical azimuthal projection $(p-q)$}

The azimuthal equal-area projection is given by

$$
\begin{aligned}
{\left[\begin{array}{c}
p \\
q
\end{array}\right] } & =\sqrt{\frac{2}{1+x_{\text {lune }}}\left[\begin{array}{l}
y_{\text {lune }} \\
z_{\text {lune }}
\end{array}\right]} \\
& =\frac{1}{\sqrt{3}}\left[\frac{\frac{-\left(\lambda_{1}-2 \lambda_{2}+\lambda_{3}\right)}{\sqrt{\lambda_{1}^{2}+\lambda_{2}^{2}+\lambda_{3}^{2}+\left(\lambda_{1}-\lambda_{3}\right) \sqrt{\left(\lambda_{1}^{2}+\lambda_{2}^{2}+\lambda_{3}^{2}\right) / 2}}}}{\sqrt{\lambda_{1}^{2}+\lambda_{2}^{2}+\lambda_{3}^{2}+\left(\lambda_{1}-\lambda_{3}\right) \sqrt{\left(\lambda_{1}^{2}+\lambda_{2}^{2}+\lambda_{3}^{2}\right) / 2}}}\right] .
\end{aligned}
$$

$\left.\begin{array}{l}\text { Since } \\ (p, q)=(\mp(\sqrt{6}-\sqrt{2}) / 2,0)\end{array}\right)$ and \pm ISO is projected at $(p, q)=(0, \pm \sqrt{2})$ in this diagram, we define the normalized coordinates $(\tilde{p}, \tilde{q})=(-p /(\sqrt{6}-\sqrt{2}), q / \sqrt{2})$.

Conversely, the azimuthal equal-area projection is

$$
\left[\begin{array}{c}
x_{\text {lune }} \\
y_{\text {lune }} \\
z_{\text {lune }}
\end{array}\right]=\frac{1}{2}\left[\begin{array}{c}
2-p^{2}-q^{2} \\
p \sqrt{4-p^{2}-q^{2}} \\
q \sqrt{4-p^{2}-q^{2}}
\end{array}\right]
$$

Substituting this into (39), we get the original coordinates

$$
\left[\begin{array}{l}
\lambda_{1} \\
\lambda_{2} \\
\lambda_{3}
\end{array}\right]=\frac{M_{o}}{2 \sqrt{3}}\left[\begin{array}{c}
\sqrt{4-p^{2}-q^{2}}(\sqrt{2} q-p)+\sqrt{3}\left(2-p^{2}-q^{2}\right) \\
\sqrt{4-p^{2}-q^{2}}(\sqrt{2} q+2 p) \\
\sqrt{4-p^{2}-q^{2}}(\sqrt{2} q-p)-\sqrt{3}\left(2-p^{2}-q^{2}\right)
\end{array}\right] .
$$

Solving the condition of $\lambda_{1}=\lambda_{2}$ or $\lambda_{2}=\lambda_{3}$, the outside boundary of this diagram is given by

$$
p= \pm \frac{\sqrt{3\left(8-3 q^{2}\right)}-\sqrt{8-q^{2}}}{4}
$$

or in other words,

$$
q= \pm \sqrt{\frac{4-5 p^{2}-\sqrt{9 p^{4}+24 p^{2}}}{2}} .
$$

Appendix 9: (i) Spherical cylindrical projection $(\gamma-\zeta)$ The coordinates on the cylinder is given by

$$
\left[\begin{array}{l}
\gamma \\
\zeta
\end{array}\right]=\left[\begin{array}{c}
\tan ^{-1}\left(y_{\text {lune }} / x_{\text {lune }}\right) \\
z_{\text {lune }}
\end{array}\right]=\left[\begin{array}{c}
-\tan ^{-1}\left(\frac{\lambda_{1}-2 \lambda_{2}+\lambda_{3}}{\sqrt{3}\left(\lambda_{1}-\lambda_{3}\right)}\right) \\
\frac{\lambda_{1}+\lambda_{2}+\lambda_{3}}{\sqrt{3\left(\lambda_{1}^{2}+\lambda_{2}^{2}+\lambda_{3}^{2}\right)}}
\end{array}\right] .
$$

The normalized coordinate system that projects \pm CLVD onto $(\tilde{\gamma}, \tilde{\zeta})=( \pm 1,0)$ is $(\tilde{\gamma}, \tilde{\zeta})=(-6 \gamma / \pi, \zeta)$.

Conversely, the point on the lune is given by

$$
\left[\begin{array}{l}
x_{\text {lune }} \\
y_{\text {lune }} \\
z_{\text {lune }}
\end{array}\right]=\left[\begin{array}{c}
\sqrt{1-\zeta^{2}} \cos \gamma \\
\sqrt{1-\zeta^{2}} \sin \gamma \\
\zeta
\end{array}\right] .
$$

Substituting this into (39), we get the original coordinates

$$
\left[\begin{array}{l}
\lambda_{1} \\
\lambda_{2} \\
\lambda_{3}
\end{array}\right]=\sqrt{2 / 3} M_{o}\left[\begin{array}{c}
\zeta-\sqrt{2} \sin \left(\gamma-\frac{\pi}{3}\right) \sqrt{1-\zeta^{2}} \\
\zeta+\sqrt{2} \sin \gamma \sqrt{1-\zeta^{2}} \\
\zeta-\sqrt{2} \sin \left(\gamma+\frac{\pi}{3}\right) \sqrt{1-\zeta^{2}}
\end{array}\right] .
$$

\section{Appendix 10: (j) Modified spherical cylindrical projection $(a-b)$}

The cylindrical projection $\gamma-\zeta(|\gamma| \leq \pi / 6,|\zeta| \leq 1)$ can be modified to a diamond shape $a-b(|a|+|b| \leq 1)$ with areal density kept constant and with any horizontal 
lines kept horizontal. The conservation of areal density is given by

$$
\int_{0}^{\zeta} \frac{\pi}{3} d \zeta / \frac{2 \pi}{3}=\int_{0}^{b} 2(1-|b|) d b / 2 \Leftrightarrow \zeta=b(2-|b|)
$$

and the horizontal coordinate is modified proportionally

$$
a=\frac{6}{\pi} \gamma(1-|b|) \text {. }
$$

Then, the forward conversion is given by

$$
\begin{aligned}
{\left[\begin{array}{l}
a \\
b
\end{array}\right] } & =\left[\begin{array}{c}
\frac{6}{\pi} \gamma \sqrt{1-|\zeta|} \\
\operatorname{sgn}(\zeta)(1-\sqrt{1-|\zeta|})
\end{array}\right] \\
& =\left[\begin{array}{c}
-\frac{6}{\pi} \tan ^{-1}\left(\frac{\lambda_{1}-2 \lambda_{2}+\lambda_{3}}{\sqrt{3}\left(\lambda_{1}-\lambda_{3}\right)}\right) \sqrt{1-\frac{\left|\lambda_{1}+\lambda_{2}+\lambda_{3}\right|}{\sqrt{3\left(\lambda_{1}^{2}+\lambda_{2}^{2}+\lambda_{3}^{2}\right)}}} \\
\frac{\left(\lambda_{1}+\lambda_{2}+\lambda_{3}\right) / \sqrt{3\left(\lambda_{1}^{2}+\lambda_{2}^{2}+\lambda_{3}^{2}\right)}}{1+\sqrt{1-\left|\lambda_{1}+\lambda_{2}+\lambda_{3}\right| / \sqrt{3\left(\lambda_{1}^{2}+\lambda_{2}^{2}+\lambda_{3}^{2}\right)}}}
\end{array}\right] .
\end{aligned}
$$

The normalized coordinate system $(\tilde{a}, \tilde{b})=(-a, b)$ projects \pm CLVD onto $(\tilde{a}, \tilde{b})=( \pm 1,0)$.

Replacing (54) with the relationship

$$
\gamma=\frac{\pi}{6} \frac{a}{1-|b|}, \quad \zeta=b(2-|b|)
$$

$$
\left[\begin{array}{l}
\lambda_{1} \\
\lambda_{2} \\
\lambda_{3}
\end{array}\right]=\frac{M_{o}}{\sqrt{3}}\left[\begin{array}{c}
\sqrt{2} \zeta+\left(-\chi+\sqrt{3\left(1-\chi^{2}\right)}\right) \sqrt{1-\zeta^{2}} \\
\sqrt{2} \zeta+2 \chi \sqrt{1-\zeta^{2}} \\
\sqrt{2} \zeta+\left(-\chi-\sqrt{3\left(1-\chi^{2}\right)}\right) \sqrt{1-\zeta^{2}}
\end{array}\right]
$$

\section{Appendix 12: (I) Percentile projection ( $\varepsilon-v)$}

The vertical axis of the percentile projection is equivalent to $v$. The horizontal axis is defined as

$$
\epsilon=\frac{\lambda_{2}-\frac{\left(\lambda_{1}+\lambda_{2}+\lambda_{3}\right)}{3}}{\max \left(\left|\lambda_{1}-\frac{\left(\lambda_{1}+\lambda_{2}+\lambda_{3}\right)}{3}\right|,\left|\lambda_{3}-\frac{\left(\lambda_{1}+\lambda_{2}+\lambda_{3}\right)}{3}\right|\right)},
$$

and we get the forward conversion

$$
\left[\begin{array}{c}
\epsilon \\
v
\end{array}\right]=\left[\begin{array}{c}
\frac{-2\left(\lambda_{1}-2 \lambda_{2}+\lambda_{3}\right)}{3\left(\lambda_{1}-\lambda_{3}\right)+\left|\lambda_{1}-2 \lambda_{2}+\lambda_{3}\right|} \\
\frac{\lambda_{1}+\lambda_{2}+\lambda_{3}}{3 \max \left(\lambda_{1},-\lambda_{3}\right)}
\end{array}\right] .
$$

Since \pm CLVD corresponds to $\epsilon=\mp 1 / 2$ in this diagram, we define the normalized coordinates $(\tilde{\epsilon}, \tilde{v})=(-2 \epsilon, v)$.

We then derive the inverse conversion. Considering that $\operatorname{sgn}(\epsilon)=-\operatorname{sgn}\left(\lambda_{1}-2 \lambda_{2}+\lambda_{3}\right)$, the first row of (64) is rewritten as

$$
(2-|\epsilon|)\left(-\lambda_{1}+2 \lambda_{2}-\lambda_{3}\right)-3 \epsilon\left(\lambda_{1}-\lambda_{3}\right)=0 .
$$

we get

$$
\left[\begin{array}{l}
\lambda_{1} \\
\lambda_{2} \\
\lambda_{3}
\end{array}\right]=\sqrt{2 / 3} M_{o}\left[\begin{array}{c}
b(2-|b|)-\sqrt{2} \sin \left(\frac{\pi}{6}\left(\frac{a}{1-|b|}-2\right)\right) \sqrt{1-b^{2}(2-|b|)^{2}} \\
b(2-|b|)+\sqrt{2} \sin \left(\frac{\pi}{6} \frac{a}{1-|b|}\right) \sqrt{1-b^{2}(2-|b|)^{2}} \\
b(2-|b|)-\sqrt{2} \sin \left(\frac{\pi}{6}\left(\frac{a}{1-|b|}+2\right)\right) \sqrt{1-b^{2}(2-|b|)^{2}}
\end{array}\right] .
$$

\section{Appendix 11: (k) Spherical cylindrical orthogonal projection $(X-\zeta)$}

The orthogonal projection of the cylinder is given by

$$
\left[\begin{array}{l}
\chi \\
\zeta
\end{array}\right]=\left[\begin{array}{l}
\sin \gamma \\
z_{\text {lune }}
\end{array}\right]=\left[\begin{array}{c}
\frac{y_{\text {lune }}}{\sqrt{1-z_{\text {lune }}^{2}}} \\
z_{\text {lune }}
\end{array}\right]=\left[\begin{array}{c}
\frac{-\left(\lambda_{1}-2 \lambda_{2}+\lambda_{3}\right) / 2}{\sqrt{\lambda_{1}^{2}+\lambda_{2}^{2}+\lambda_{3}^{2}-\lambda_{1} \lambda_{2}-\lambda_{2} \lambda_{3}-\lambda_{1} \lambda_{3}}} \\
\frac{\lambda_{1}+\lambda_{2}+\lambda_{3}}{\sqrt{3\left(\lambda_{1}^{2}+\lambda_{2}^{2}+\lambda_{3}^{2}\right)}}
\end{array}\right] .
$$

The normalized coordinate system $(\tilde{\chi}, \tilde{\zeta})=(-2 \chi, \zeta)$ projects $\pm \operatorname{CLVD}$ onto $(\tilde{\chi}, \tilde{\zeta})=( \pm 1,0)$.

Conversely, the point on the lune is written as

$$
\left[\begin{array}{l}
x_{\text {lune }} \\
y_{\text {lune }} \\
z_{\text {lune }}
\end{array}\right]=\left[\begin{array}{c}
\sqrt{1-\chi^{2}} \sqrt{1-\zeta^{2}} \\
\chi \sqrt{1-\zeta^{2}} \\
\zeta
\end{array}\right]
$$

Inserting this equation into (39), we get the original coordinates
The second row of (64) is rewritten as

$$
\left\{\begin{array}{l}
\lambda_{1}+\lambda_{2}+\lambda_{3}-3 v \lambda_{1}=0 \quad \lambda_{1}+\lambda_{3} \geq 0 \\
\lambda_{1}+\lambda_{2}+\lambda_{3}+3 v \lambda_{1}=0 \quad \lambda_{1}+\lambda_{3} \leq 0
\end{array} .\right.
$$

The combination of (65) and (66) results in

$$
\left[\begin{array}{l}
\lambda_{1} \\
\lambda_{2} \\
\lambda_{3}
\end{array}\right] \propto\left\{\begin{array}{c}
2-|\epsilon|-\epsilon \\
(2-|\epsilon|) \nu+2 \epsilon-3 v \epsilon \\
(2 v-1)(2-|\epsilon|)-\epsilon \\
(2 v+1)(2-|\epsilon|)-\epsilon \\
(2-|\epsilon|) \nu+2 \epsilon+3 v \epsilon \\
-2+|\epsilon|-\epsilon
\end{array}\right] \quad \lambda_{1}+\lambda_{3} \geq 0
$$

Considering $\lambda_{1}+\lambda_{3} \lesseqgtr 0 \Leftrightarrow v(2-|\epsilon|)-\epsilon \lesseqgtr 0$, we get the inverse conversion

$$
\left[\begin{array}{l}
\lambda_{1} \\
\lambda_{2} \\
\lambda_{3}
\end{array}\right] \propto\left[\begin{array}{c}
(2-|\epsilon|)(v+1)-\epsilon-\operatorname{sgn}(v(2-|\epsilon|)-\epsilon) v(2-|\epsilon|) \\
(2-|\epsilon|) v+2 \epsilon-\operatorname{sgn}(v(2-|\epsilon|)-\epsilon) 3 v \epsilon \\
(2-|\epsilon|)(v-1)-\epsilon+\operatorname{sgn}(v(2-|\epsilon|)-\epsilon) v(2-|\epsilon|)
\end{array}\right] .
$$


The sharp interface corresponding to $\max \left(\lambda_{1},-\lambda_{3}\right)$ is given by $\left|\lambda_{1}+\lambda_{3}\right|=0$, which results in

$$
v=\frac{\epsilon}{2-|\epsilon|} \text {. }
$$

\section{Appendix 13: (m) Modified percentile projection} $(c-v)$

Applying $c=2 \epsilon(1-|v|)$ to (64), we get

$$
\left[\begin{array}{c}
c \\
v
\end{array}\right]=\left[\begin{array}{c}
\frac{-4\left(\lambda_{1}-2 \lambda_{2}+\lambda_{3}\right)}{3\left(\lambda_{1}-\lambda_{3}\right)+\left|\lambda_{1}-2 \lambda_{2}+\lambda_{3}\right|}\left(1-\frac{\left|\lambda_{1}+\lambda_{2}+\lambda_{3}\right|}{3 \max \left(\lambda_{1},-\lambda_{3}\right)}\right) \\
\frac{\lambda_{1}+\lambda_{2}+\lambda_{3}}{3 \max \left(\lambda_{1},-\lambda_{3}\right)}
\end{array}\right] .
$$

The normalized coordinates in which \pm CLVD corresponds to $(\tilde{c}, \tilde{v})=( \pm 1,0)$ are $(\tilde{c}, \tilde{v})=(-c, v)$.

Applying $\epsilon=c / 2(1-|v|)$ to (68), we get the inverse relationship

$$
\begin{aligned}
& {\left[\begin{array}{l}
\lambda_{1} \\
\lambda_{2} \\
\lambda_{3}
\end{array}\right] \propto\left[\begin{array}{c}
(4(1-|v|)-|c|)(v+1)-c \\
(4(1-|v|)-|c|) v+2 c \\
(4(1-|v|)-|c|)(v-1)-c
\end{array}\right]} \\
& -\operatorname{sgn}(4 v(1-|v|)-v|c|-c)\left[\begin{array}{c}
4 v(1-|v|)-v|c| \\
3 v c \\
-4 v(1-|v|)+v|c|
\end{array}\right] .
\end{aligned}
$$

The sharp interface corresponding to $\max \left(\lambda_{1},-\lambda_{3}\right)$ is given by $\left|\lambda_{1}+\lambda_{3}\right|=0$; therefore,

$$
v(4(1-|v|)-|c|)-c=0 .
$$

Using the relationship of $\operatorname{sgn}(v)=\operatorname{sgn}(c)$ under this condition, we get

$$
v=\operatorname{sgn}(c) \frac{4-|c|-\sqrt{16-24|c|+c^{2}}}{8} .
$$

Received: 8 December 2015 Accepted: 25 February 2016 Published online: 31 March 2016

\section{References}

Adamová P, Š́lený J (2010) Non-double-couple earthquake mechanism as an artifact of the point-source approach applied to a finite-extent focus. Bull Seismol Soc Am 100(2):447-457. doi:10.1785/0120090097

Ampuero J-P, Dahlen FA (2005) Ambiguity of the moment tensor. Bull Seismol Soc Am 95(2):390-400. doi:10.1785/0120040103

Aso N, Ide S (2014) Focal mechanisms of deep low-frequency earthquakes in Eastern Shimane in Western Japan. J Geophys Res 119(1):364-377. doi:10.1002/2013JB010681

Aso N, Tsai VC (2014) Cooling magma model for deep volcanic long-period earthquakes. J Geophys Res 119(11):8442-8456. doi:10.1002/2014JB011180

Bowers D, Hudson JA (1999) Defining the scalar moment of a seismic source with a general moment tensor. Bull Seismol Soc Am 89(5):1390-1394

Chapman CH, Leaney WS (2012) A new moment-tensor decomposition for seismic events in anisotropic media. Geophys J Int 188(1):343-370. doi:10.1111/j.1365-246X.2011.05265.x
Dufumier $H$, Rivera $L$ (1997) On the resolution of the isotropic component in moment tensor. Geophys J Int 131(3):595-606. doi:10.1111/j.1365246X.1997.tb06601.x

Dziewonski AM, Gilbert F (1974) Temporal variation of the seismic moment tensor and the evidence of precursive compression for two deep earthquakes. Nature 247:185-188. doi:10.1038/247185a0

Ekström G (1994) Anomalous earthquakes on volcano ringfault structures. Earth Planet Sci Lett 128(3-4):707-712. doi:10.1016/0012-821X(94)90184-8

Ford SR, Dreger DS, Walter WR (2008) Source characterization of the 6 August 2007 Crandall Canyon Mine seismic event in central Utah. Seismol Res Lett 79(5):637-644. doi:10.1785/gssrl.79.5.637

Ford SR, Dreger DS, Walter WR (2009) Identifying isotropic events using a regional moment tensor inversion. J Geophys Res 114(B1):B01306. doi:10.1029/2008JB005743

Ford SR, Dreger DS, Walter WR (2010) Network sensitivity solutions for regional moment-tensor inversions. Bull Seismol Soc Am 100(5A):1962-1970. doi:10.1785/0120090140

Foulger GR, Julian BR, Hill DP, Pitt AM, Malin PE, Shalev E (2004) Non-doublecouple microearthquakes at Long Valley caldera, California, provide evidence for hydraulic fracturing. J Volcanol Geotherm Res 132(1):45-71. doi:10.1016/S0377-0273(03)00420-7

Frohlich C (1992) Triangle diagrams: ternary graphs to display similarity and diversity of earthquake focal mechanisms. Phys Earth Planet Int 75(1):193-198. doi:10.1016/0031-9201(92)90130-N

Hara T, Kuge K, Kawakatsu H (1995) Determination of the isotropic component of the 1994 Bolivia deep earthquake. Geophys Res Lett 22(16):2265-2268. doi:10.1029/95GL01602

Honda H, Emura K (1958) Some charts for studying the mechanism of earthquakes. Sci Rep Tohoku Univ Ser 5 Geophys 9(3):113-119

Hudson JA, Pearce RG, Rogers RM (1989) Source type plot for inversion of the moment tensor. J Geophys Res 94(B1):765-774. doi:10.1029/ JB094iB01 p00765

Iguchi M (1994) A vertical expansion source model for the mechanisms of earthquakes originated in the magma conduit of an andesitic volcano: Sakurajima, Japan. Bull Volcanol Soc Jpn 39(2):49-67

Julian BR, Miller AD, Foulger GR (1997) Non-double-couple earthquake mechanisms at the Hengill-Grensdalur volcanic complex, southwest Iceland. Geophys Res Lett 24(7):743-746. doi:10.1029/97GL00499

Julian BR, Miller AD, Foulger GR (1998) Non-double-couple earthquakes 1: theory. Rev Geophys 36(4):525-549. doi:10.1029/98RG00716

Kawakatsu H (1989) Centroid single force inversion of seismic waves generated by landslides. J Geophys Res 94(B9):12363-12374. doi:10.1029/ JB094iB09p12363

Kawakatsu H, Yamamoto M (2007) Volcano seismology. In: Kanamori H, Schubert G (eds) Earthquake seismology. Treatise on geophysics, vol 4 Elsevier, New York, pp 389-420. doi:10.1016/B978-044452748-6.00073-0

Kikuchi M, Kanamori H, Satake K (1993) Source complexity of the 1988 Armenian Earthquake: evidence for a slow after-slip event. J Geophys Res 98(B9):15797-15808. doi:10.1029/93JB01568

Knopoff L, Randall MJ (1970) The compensated linear-vector dipole: a possible mechanism for deep earthquakes. J Geophys Res 75(26):4957-4963. doi:10.1029/JB075i026p04957

Kuge K, Kawakatsu H (1990) Analysis of a deep non-double couple earthquake using very broadband data. Geophys Res Lett 17(3):227-230. doi:10.1029/ GL017i003p00227

Kuge K, Kawakatsu H (1993) Significance of non-double couple components of deep and intermediate-depth earthquakes: implications from moment tensor inversions of long-period seismic waves. Phys Earth Planet Int 75(4):243-266. doi:10.1016/0031-9201(93)90004-S

Kuge K, Lay T (1994a) Data-dependent non-double-couple components of shallow earthquake source mechanisms: effects of waveform instability. Geophys Res Lett 21(1):9-12. doi:10.1029/93GL03294

Kuge K, Lay T (1994b) Systematic non-double-couple components of earthquake mechanisms: the role of fault zone irregularity. J Geophys Res 99(B8):15457-15467. doi:10.1029/94JB00140

Legrand D, Kaneshima S, Kawakatsu H (2000) Moment tensor analysis of nearfield broadband waveforms observed at Aso Volcano, Japan. J Volcanol Geotherm Res 101(1-2):155-169. doi:10.1016/\$0377-0273(00)00167-0 
Miller AD, Foulger GR, Julian BR (1998) Non-double-couple earthquakes 2: observations. Rev Geophys 36(4):551-568. doi:10.1029/98RG00717

Minson SE, Dreger DS, Bürgmann R, Kanamori H, Larson KM (2007) Seismically and geodetically determined nondouble-couple source mechanisms from the 2000 Miyakejima volcanic earthquake swarm. J Geophys Res 112(B10):B10308. doi:10.1029/2006JB004847

Nakamichi H, Hamaguchi H, Tanaka S, Ueki S, Nishimura T, Hasegawa A (2003) Source mechanisms of deep and intermediate-depth low-frequency earthquakes beneath Iwate volcano, northeastern Japan. Geophys J Int 154(3):811-828. doi:10.1046/j.1365-246X.2003.01991.x

Nayak A, Dreger DS (2015) Source-type-specific inversion of moment tensors. Bull Seismol Soc Am 105(6):2987-3000. doi:10.1785/0120140334

Nishidomi I, Takeo M (1996) Seismicity and a focal mechanism of low-frequency earthquakes occurring in the western part of Tochigi prefecture, Japan. Bull Volcanol Soc Jpn 41(2):43-59 (in Japanese with English

\section{abstract)}

Okada T, Hasegawa A (2000) Activity of deep low-frequency microearthquakes and their moment tensors in northeastern Japan. Bull Volcanol Soc Jpn 45(2):47-63 (in Japanese with English abstract)

Okal EA, Geller RJ (1979) On the observability of isotropic seismic sources: the July 31, 1970 Colombian earthquake. Phys Earth Planet Inter 18(3):176196. doi:10.1016/0031-9201(79)90113-4

Ross A, Foulger GR, Julian BR (1996) Non-double-couple earthquake mechanisms at the Geysers geothermal area, California. Geophys Res Lett 23(8):877-880. doi:10.1029/96GL00590

Ross A, Foulger GR, Julian BR (1999) Source processes of industrially-induced earthquakes at the Geysers geothermal area, California. Geophysics 64(6):1877-1889. doi:10.1190/1.1444694

Saraò A, Panza G, Privitera E, Cocina O (2001) Non-double-couple mechanisms in the seismicity preceding 1991-1993 Etna volcano eruption. Geophys J Int 145(2):319-335. doi:10.1046/j.1365-246x.2001.01375.x

Shearer PM (2009) Introduction to seismology. Cambridge University Press, Cambridge

Shuler A, Nettles M, Ekström G (2013) Global observation of vertical-CLVD earthquakes at active volcanoes. J Geophys Res 118(1):138-164. doi:10.1 029/2012JB009721
Sipkin SA (1986) Interpretation of non-double-couple earthquake mechanisms derived from moment tensor inversion. J Geophys Res 91(B1):531547. doi:10.1029/JB091iB01p00531

Tape W, Tape C (2012) A geometric comparison of source-type plots for moment tensors. Geophys J Int 190(1):499-510. doi:10.1111/j.1365-246X.2012.05490.x

Tape W, Tape C (2013) The classical model for moment tensors. Geophys J Int 195(3):1701-1720. doi:10.1093/gji/ggt302

Tape W, Tape C (2015) A uniform parametrization of moment tensors. Geophys J Int 202(3):2074-2081. doi:10.1093/gji/ggv262

Vavryčuk V (2001) Inversion for parameters of tensile earthquakes. J Geophys Res 106(B8):16339-16355. doi:10.1029/2001JB000372

Vavryčuk V (2002) Non-double-couple earthquakes of 1997 January in West Bohemia, Czech Republic: evidence of tensile faulting. Geophys J Int 149(2):364-373. doi:10.1046/j.1365-246X.2002.01654.x

Vavryčuk V (2005) Focal mechanisms in anisotropic media. Geophys J Int 161(2):334-346. doi:10.1111/j.1365-246X.2005.02585.x

Vavryčuk V (2013) Is the seismic moment tensor ambiguous at a material interface? Geophys J Int 194(1):395-400. doi:10.1093/gji/ggt084

Vavryčuk V (2015) Moment tensor decompositions revisited. J Seismol 19(1):231-252. doi:10.1007/s10950-014-9463-y

Vavryčuk V, Bohnhoff M, Jechumtálová Z, Kolář P, Šílený J (2008) Non-doublecouple mechanisms of microearthquakes induced during the 2000 injection experiment at the KTB site, Germany: a result of tensile faulting or anisotropy of a rock? Tectonophysics 456(1):74-93. doi:10.1016/j. tecto.2007.08.019

Walter F, Clinton JF, Deichmann N, Dreger DS, Minson SE, Funk M (2009) Moment tensor inversions of icequakes on Gornergletscher, Switzerland. Bull Seismol Soc Am 99(2A):852-870. doi:10.1785/0120080110

Zhu L, Ben-Zion Y (2013) Parameterization of general seismic potency and moment tensors for source inversion of seismic waveform data. Geophys J Int 194(2):839-843. doi:10.1093/gji/ggt137

\section{Submit your manuscript to a SpringerOpen ${ }^{\odot}$ journal and benefit from:}

- Convenient online submission

- Rigorous peer review

- Immediate publication on acceptance

- Open access: articles freely available online

- High visibility within the field

- Retaining the copyright to your article

Submit your next manuscript at $>$ springeropen.com 